\title{
One-dimensional heterogeneous solids with uncertain elastic modulus in presence of long-range interactions: Interval versus stochastic analysis
}

\author{
Giuseppe Muscolino ${ }^{\mathrm{a}, \mathrm{d}, *}$, Alba Sofi ${ }^{\mathrm{b}, \mathrm{d}}$, Massimiliano Zingales ${ }^{\mathrm{c}, \mathrm{e}}$ \\ a Dipartimento di Ingegneria Civile, Informatica, Edile, Ambientale e Matematica Applicata, University of Messina, Villaggio S. Agata, 98166 Messina, Italy

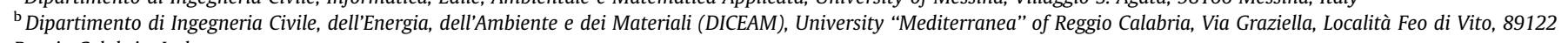 \\ Reggio Calabria, Italy \\ ${ }^{\mathrm{c}}$ Dipartimento di Ingegneria Civile, Ambientale ed Aerospaziale (DICA), University of Palermo, Viale delle Scienze ed.8, 90128 Palermo, Italy \\ ${ }^{\mathrm{d}}$ Interuniversity Centre of Structural, Theoretical and Experimental Dynamics (C.I.Di.S.), Universities of Palermo, Messina, Reggio Calabria and Enna "Kore", Italy \\ e Dipartimento di Biomeccanica e Biomateriali, Istituto Euro-Mediterraneo di Scienza e Tecnologia, I.E.Me.S.T., Via Emerico Amari n.128, 90100 Palermo, Italy
}

\section{A R T I C L E I N F O}

\section{Article history:}

Received 31 May 2012

Accepted 8 March 2013

Available online 8 April 2013

\section{Keywords:}

Non-local elasticity

Interval field

Random field

Karhunen-Loève decomposition

Upper bound and lower bound

Response statistics

\begin{abstract}
A B S T R A C T
The analysis of one-dimensional non-local elastic solids with uncertain Young's modulus is addressed. Non-local effects are represented as long-range central body forces between non-adjacent volume elements. For comparison purpose, the fluctuating elastic modulus of the material is modeled following both a probabilistic and a non-probabilistic approach. To this aim, a novel definition of the interval field concept, able to limit the overestimation affecting ordinary interval analysis, is introduced. Approximate closed-form expressions are derived for the bounds of the interval displacement field as well as for the mean-value and variance of the stochastic response.
\end{abstract}

(ㄷ) 2013 Elsevier Ltd. All rights reserved.

\section{Introduction}

Almost all structural systems exhibit physical and geometrical uncertainties due to modeling inaccuracies, measurement and manufacturing errors or other factors. These sources of uncertainty are usually described following two contrasting points of view, known as probabilistic and non-probabilistic approaches. Probabilistic approaches represent the uncertain parameters as random variables or random fields with given probability density function $[1,2]$. Criticism on the credibility of these approaches arises when they are based on limited data [3]. If available information on the uncertain parameters is fragmentary or incomplete, non-probabilistic approaches [4], such as convex models, interval models and fuzzy sets [5], can be alternatively applied. The interval model turns out to be a very useful tool when available data provide accurate information on the range within which a non-deterministic structural property may vary. Indeed, this model is derived from the interval analysis [6-8] in which the number is treated as an interval

\footnotetext{
* Corresponding author at: Dipartimento di Ingegneria Civile, Informatica, Edile, Ambientale e Matematica Applicata, University of Messina, Villaggio S. Agata, 98166 Messina, Italy. Tel.: +39090 397 7159; fax: +39090 3977480.

E-mail addresses: gmuscolino@unime.it (G. Muscolino), alba.sofi@unirc.it (A. Sofi), massimiliano.zingales@unipa.it (M. Zingales).
}

variable ranging between its lower and upper bounds. In spite of the simplicity of the interval concept, the application of the interval analysis to practical engineering problems often leads to very complex algorithms. For this reason, in the literature several approximate methods have been proposed to perform both the static and dynamic analysis of structures with interval uncertainties [9-15]. Moreover, the "ordinary" interval analysis [6] suffers from two main shortcomings which hinder its application to structural engineering problems: the first one is the so-called dependency phenomenon; the second one is the inability to handle spatial dependency of a model property. The latter problem involves the introduction of the so-called interval field $[16,17]$ as a proper extension of the random field concept within the non-probabilistic framework. On the other hand, the dependency phenomenon often leads to an overestimation of the interval solution width that could be catastrophic from an engineering point of view $[3,8,18]$. This occurs when an expression contains multiple instances of one or more interval variables.

Both probabilistic and non-probabilistic models have been widely used in the literature to investigate the effects of uncertainties in the context of the classical local continuum models. However, nowadays it is recognized that classical local continuum mechanics that is an intrinsically scale-free theory fails to predict several phenomena, such as screw dislocation, dispersion of elastic 
waves, stress tip concentration, where long-range intermolecular forces and microstructure play a significant role. A considerable research effort has been devoted since the sixties of the last century (see e.g. [19-21]) to develop non-local models of elastic solids aiming to capture, in a continuum field formulation, the influence of the inner microstructure of the matter. Some of these models involve the introduction of additional contributions in the stressstrain relations of materials in terms of integrals (see e.g. $[22,23]$ ) or gradients (see e.g. $[24,25]$ ) of the strain field, yielding the socalled integral or weak and gradient or strong theories of non-local elasticity. A quite different description of non-local effects as longrange body forces, referred to as mechanically-based model of nonlocal elasticity theory, has been recently proposed [26-29].

The random microstructure of materials has been analyzed in the context of weak and strong models of non-local elasticity at the beginning of the century [30,31]. Relying on the mechanically-based approach, a random model of long-range interactions in 1D heterogeneous solids with uncertain mass density has been recently developed [32].

In this paper, the effects of Young's modulus uncertainty on the response of $1 \mathrm{D}$ heterogeneous solids with long-range interactions subjected to static loads are analyzed. Long-range interactions are handled resorting to the mechanically-based model of non-local elasticity. The fluctuations of the uncertain material property along the 1D solid are modelled by adopting both a non-probabilistic and a probabilistic approach, namely both as an interval field and a homogeneous random field. An efficient procedure is proposed to characterize the interval and random displacement fields describing the response of the 1D non-local solid with uncertainbut-bounded and randomly varying Young's modulus, respectively. The following key steps are involved: (i) the use of the so-called improved interval analysis presented in Ref. [15] in order to limit the effects of the dependency phenomenon; (ii) the introduction of a novel definition of the interval field able to describe the dependency between values of the fluctuating elastic modulus at various abscissas along the 1D solid; (iii) the finite difference discretization of the governing equations; (iv) the evaluation in explicit approximate form of the lower and upper bounds of the interval displacement field for the 1D non-local solid with interval Young's modulus; ( $v$ ) the derivation of approximate closed-form expressions of the mean-value vector and covariance matrix of the stochastic response for the 1D non-local solid with randomly varying elastic modulus.

The main novelties introduced in the present study may be summarized as follows: (i) the use of the interval model to describe material property uncertainty in 1D non-local elastic solids; (ii) a novel definition of the interval field, quite different from existing ones $[16,17]$, in order to account for the spatial dependency of the uncertain-but-bounded material property; (iii) the evaluation in explicit approximate form of the bounds and statistics of the response within the context of the interval and random models of the uncertain Young's modulus, respectively.

Numerical results concerning a non-local elastic bar under tension with uncertain Young's modulus of the material are presented to demonstrate the effectiveness of the proposed procedure. The consistency of the novel interval field definition is also scrutinized through appropriate comparisons between the interval and stochastic responses.

\section{The long-range interaction model in a one-dimensional heterogeneous solid}

In this section, the mechanically-based model of $1 \mathrm{D}$ solids with long-range interactions, recently proposed by Di Paola et al. [26$29]$, is briefly summarized. To this aim, let us consider a 1D elastic bar of length $L$ referred to a coordinate system $0-x$ positive rightward (Fig. 1a).

In the context of the mechanically-based model of non-local elasticity, it is thought that the actions applied to a volume element $d V(x)$ at the abscissa $x$ consist of three contributions: the well-known local Cauchy stress, $\sigma^{(l)}(x)$, the external body force field, $b(x)$, and the additional central body forces exerted by nonadjacent volumes $d V(\xi)$ located at the abscissas $\xi$ (Fig. 1b). Moreover, it is assumed that the long-range interactions between volumes $d V(x)$ and $d V(\xi)$ depend on the product of the elementary interacting masses, $d M(x)=\rho(x) d V(x)$ and $d M(\xi)=\rho(\xi) d V(\xi), \rho(x)$ and $\rho(\xi)$ being the mass density of the material at locations $x$ and $\xi$, as well as on their relative axial displacement field $\eta(x, \xi)=u(\xi)-u(x)$ (see Fig. 1b), i.e.:

$q(x, \xi) d M(x) d M(\xi)=c_{q} \rho^{2} A(x) A(\xi) g(x, \xi) \eta(x, \xi) d x d \xi$

where $q(x, \xi)=c_{q} g(x, \xi) \eta(x, \xi)$ is the specific long-range force; $\left[c_{q}\right]=$ $F / L M^{2}$ is a physical material-dependent force constant; $A(x)$ is the cross-section at the abscissa $x ; \rho=\rho(x)=\rho(\xi)$ denotes the mass density of the material herein assumed constant along the bar; $g(x, \xi)$ is a material-dependent, symmetric, real-valued scalar function which must be strictly positive to satisfy the Drucker stability criterion. Moreover, the function $g(x, \xi)$ is monotonically decreasing with the distance $|x-\xi|$ between interacting volume elements.

Then, the equilibrium equation of the 1D heterogeneous solid with long-range interactions takes the following form [29,32]:

$$
\begin{aligned}
& \frac{d}{d x}\left[E^{*}(x) A(x) \frac{d u(x)}{d x}\right]+c_{q} \rho^{2} A(x) \int_{0}^{L} A(\xi) g(x, \xi) \eta(x, \xi) d \xi \\
& \quad=-A(x) b(x)
\end{aligned}
$$

where $E^{*}(x)$ is a non-local elastic modulus, related to the measure of the Young's modulus of the material $E(x)$ as $E^{*}(x)=E(x) \beta_{1}$, with $0 \leqslant \beta_{1} \leqslant 1$ being a dimensionless real coefficient which weights the amount of local effects. The non-local elastic modulus $E^{*}(x)$ represents the value of the elastic modulus measured in a specimen at a sufficient distance from the boundaries that any edge effect may be disregarded.

Finally, the boundary conditions associated to the integro-differential equation in Eq. (2) read:

$$
\begin{aligned}
& u(0)=u_{0} \text { or }\left.E^{*}(x) A(x) \frac{d u(x)}{d x}\right|_{x=0}=-F_{0} \\
& u(L)=u_{L} \text { or }\left.E^{*}(x) A(x) \frac{d u(x)}{d x}\right|_{x=L}=F_{L}
\end{aligned}
$$

where $u_{i}$ and $F_{i}(i=0, L)$ are prescribed displacements and loads at $x=0$ and $x=L$, respectively.

A remarkable feature of the mechanically-based model of non-local elasticity is that the static boundary conditions in Eqs. (3a,b) involve only the local Cauchy stress. Indeed, the non-local effects, being represented by long-range body forces, vanish at the edges of the body where the applied external tractions are equilibrated only by the contact Cauchy stress (see e.g. $[27,28])$. Moreover, by performing a finite difference discretization of Eq. (2), the physical implications of the mechanicallybased model of non-local elasticity can be captured, as will be outlined next.

The aim of the present study is to analyze the effects of the Young's modulus uncertainty on the non-local displacement field of the 1D heterogeneous solid with long-range interactions ruled by Eq. (2). For comparison purpose, in the next sections both the well-known probabilistic approach and the interval model will be adopted to represent the uncertain material property. 
(a)
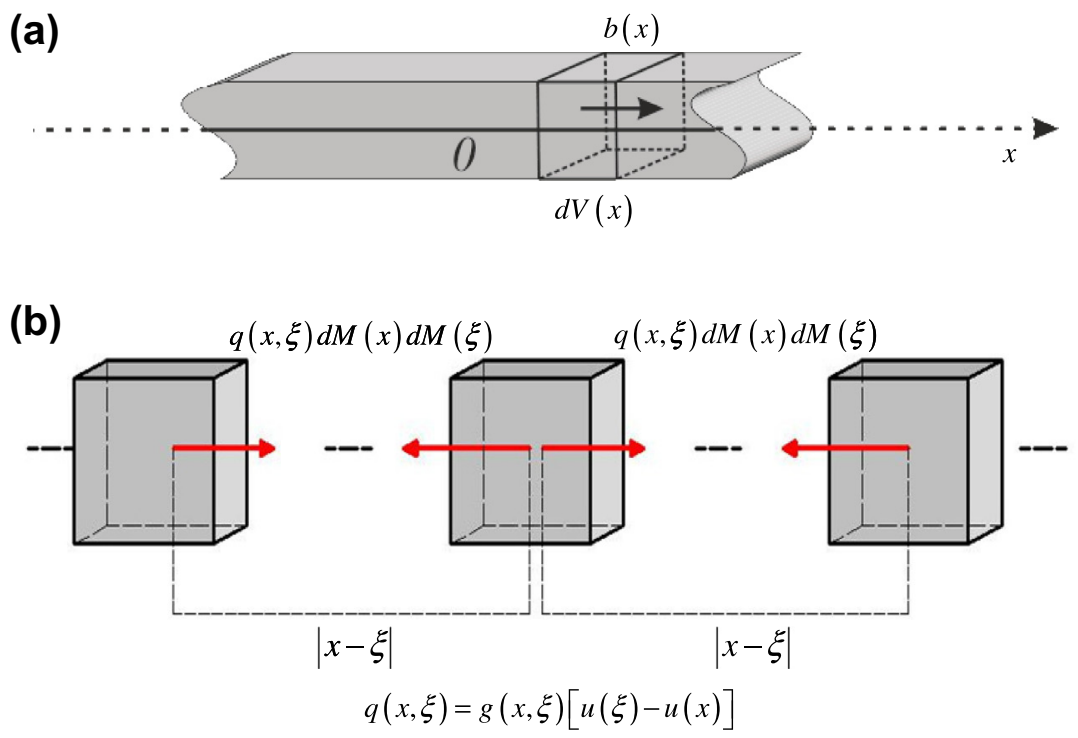

Fig. 1. (a) One-dimensional elastic solid; (b) mechanical representation of long-range interactions.

\section{Interval model of uncertainty}

\subsection{Improved interval analysis}

Following a non-probabilistic approach, the Young's modulus of the material $E^{*}(x)$, at the generic abscissa $x$ of the 1D heterogeneous solid with long-range interactions (see Eq. (2)), is modelled here as a variable which can assume real values inside a real interval.

The interval model of uncertainty, stemming from the interval analysis $[6,8]$, turns out to be very suitable when the range of variability of a structural parameter is known (see Appendix A). Denoting by $\square \mathbb{R}$ the set of all real interval numbers, the bounded interval of real numbers $\alpha^{I} \triangleq[\underline{\alpha}, \bar{\alpha}] \in \mathbb{R}$ such that $\underline{\alpha} \leqslant \alpha \leqslant \bar{\alpha}$, can be introduced. The symbols $\underline{\alpha}$ and $\bar{\alpha}$ are the lower bound and upper bound of the interval, respectively, while the apex I characterizes the interval variables. Mathematical derivations involving real numbers $\alpha$ bounded by intervals should be performed by means of the "ordinary" interval analysis [6]. Unfortunately, the "ordinary" interval analysis suffers from the so-called dependency phenomenon, $[3,8,18]$ which often introduces a high amount of conservatism leading to useless results for real sized structures. This is due to the inability of ordinary interval arithmetic to keep track of the dependency between interval variables. Therefore, when an expression contains multiple instances of one or more interval variables, the operand interval numbers are erroneously treated as independent. When the operands are partially dependent on each other, not all combinations of values in the given intervals will be valid and the exact result interval will generally be smaller than the one produced by the formulas. In an attempt to limit the catastrophic effects of the dependency phenomenon, the generalized interval analysis [33] and the affine arithmetic [34,35] have been introduced in the literature. In these formulations, each intermediate result is represented by a linear function with a small remainder interval [36]. In the context of the stochastic analysis of structures with uncertain-but-bounded parameters, following the philosophy of the affine arithmetic, Muscolino and Sofi [15] proposed an improved interval analysis based on the definition of the so-called extra symmetric unitary interval (EUI) variable $\hat{e}_{i}^{I} \triangleq[-1,+1]$. The subscript $i$ in the interval variable $\hat{e}_{i}^{I}$ indicates that the variable is associated to the $i$ th uncertain-but-bounded parameter. Moreover, unlike the unitary interval used in the "or- dinary" interval analysis (see Appendix B), the EUI is defined in such a way that the following properties hold:

$$
\begin{aligned}
& \hat{e}_{i}^{I}-\hat{e}_{i}^{I}= {[0,0] ; \quad \hat{e}_{i}^{I} \times \hat{e}_{i}^{I}=\left(\hat{e}_{i}^{I}\right)^{2}=[1,1] ; } \\
& \hat{e}_{i}^{I} \times \hat{e}_{j}^{I}=\hat{e}_{i j}^{I}=[-1,+1], \\
& i \neq j ; \hat{e}_{i}^{I} / \hat{e}_{i}^{I}=[1,1] ; \\
& x_{i} \hat{e}_{i}^{I} \pm y_{i} \hat{e}_{i}^{I}=\left(x_{i} \pm y_{i}\right) \hat{e}_{i}^{I} ; \quad x_{i} \hat{e}_{i}^{I} \times y_{i} \hat{e}_{i}^{I}=x_{i} y_{i}\left(\hat{e}_{i}^{I}\right)^{2}=x_{i} y_{i}[1,1] .
\end{aligned}
$$

In these equations, $[1,1]=1$ is the so-called unitary thin interval. It is recalled that a thin interval occurs when $\underline{\alpha}=\bar{\alpha}$ and it is defined as $\alpha^{I} \triangleq[\underline{\alpha}, \underline{\alpha}]$, so that $\alpha \in \mathbb{R}$.

Then, in the context of the improved interval analysis, the generic interval variable $\alpha^{I}$ can be expressed in the so-called affine form, i.e.:

$\alpha^{I}=\alpha_{0}+\Delta \alpha \hat{e}_{\alpha}^{I}$

where $\hat{e}_{\alpha}^{I}$ is the EUI variable associated to $\alpha^{I}$

$\alpha_{0}=\frac{1}{2}(\underline{\alpha}+\bar{\alpha}) ; \quad \Delta \alpha=\frac{1}{2}(\bar{\alpha}-\underline{\alpha})$

denote the midpoint and the deviation of $\alpha^{I}$, respectively.

\subsection{Interval field}

Two extreme approaches are commonly used to model uncertainties within both a probabilistic and non-probabilistic framework. The first one assumes that an uncertain parameter can be represented as a single (random or interval) variable constant over the whole domain. In the second approach, a (random or interval) variable is introduced for each element of a finite element model or a dicretized system. It is argued that these two approaches are both unrealistic since they imply total spatial dependency and spatially independency of the uncertain parameter, respectively. Moreover, introducing an uncertain parameter for each element may involve heavy computations.

As known, within a probabilistic context, the spatial dependency of uncertainties is handled by the random field concept which may be viewed as an intermediate model between the two extreme approaches discussed above. While interval variables are extensively used as the non-probabilistic counterpart of random variables, handling spatial dependency is still a main 
challenge in the interval analysis of structural systems. In order to cope with this problem which hinders the application of the "ordinary" interval analysis to structural engineering problems, Moens et al. [16] introduced the concept of interval field. In analogy with the random field, an interval field is conceived as a more realistic model of interval uncertainties able to define a form of dependency between adjacent values that cannot differ as much as values that are further apart. Physically, an interval field describes an uncertain model parameter which exhibits a spatially dependent variability bounded by lower and upper values. Typical examples are uncertain material properties or loads which possess a spatial character, namely they take different values over a given domain within the same realization.

In order to gain further insight into this concept, let us consider the case in which the variability of the uncertain elastic modulus along the 1D non-local solid introduced in Section 2 is represented by the following interval function:

$E^{* I}(x)=\left[\underline{E}^{*}(x), \bar{E}^{*}(x)\right]$

where $\underline{E}^{*}(x)$ and $\bar{E}^{*}(x)$ are the lower bound and upper bound of the function $E^{*}(x)$ for every $x \in \mathbb{R}$ within the domain $[0, L]$. Without loss of generality, it is assumed that the interval function $E^{* I}(x)$ can be defined as follows:

$E^{* I}(x)=E_{0}^{*}\left[1+B^{I}(x)\right], \quad x \in[0, L]$

with midpoint $E_{0}^{*} \in \mathbb{R}$ taken constant over the whole domain $[0, L]$ and deviation $\Delta E^{*}(x)$ given, respectively, by:

$\operatorname{mid}\left\{E^{* I}(x)\right\}=\frac{\bar{E}^{*}(x)+\underline{E}^{*}(x)}{2} \equiv E_{0}^{*} ; \quad \Delta E^{*}(x)=\frac{\bar{E}^{*}(x)-\underline{E}^{*}(x)}{2}, \quad x \in[0, L]$.

In the previous equation, $\operatorname{mid}\{\cdot\}$ denotes the midpoint of the interval quantity between curly parentheses.

Furthermore, in Eq. (8) $B^{I}(x)=[\underline{B}(x), \bar{B}(x)]$ denotes a dimensionless interval function having zero midpoint and deviation $\Delta B(x) \ll 1$, i.e.:

$\operatorname{mid}\left\{B^{I}(x)\right\}=\frac{\bar{B}(x)+\underline{B}(x)}{2}=0 ;$

$\Delta B(x)=\frac{\bar{B}(x)-\underline{B}(x)}{2} \equiv \frac{\Delta E^{*}(x)}{E_{0}^{*}}=\frac{\bar{E}^{*}(x)-\underline{E}^{*}(x)}{2 E_{0}^{*}}, \quad x \in[0, L]$.

The value of the dimensionless interval function $B^{I}(x)$, at the generic abscissa $x$, is partially dependent on the values it takes at the other abscissas $\xi$ different from $x$. The key issue is to assume an appropriate pattern for modelling the spatial dependency of the interval function $B^{I}(x)$. Such spatial dependency is assumed here to be governed by a real deterministic symmetric non-negative function, $\Gamma_{B}(x, \xi)$, defined as:

$\Gamma_{B}(x, \xi)=\operatorname{mid}\left\{B^{I}(x) B^{I}(\xi)\right\} \equiv \frac{\operatorname{mid}\left(E^{* I}(x) E^{* I}(\xi)\right)}{\left(E_{0}^{*}\right)^{2}}-1, \quad x, \xi \in[0, L]$.

Notice that $\Gamma_{B}(x, \xi)$ represents the midpoint of the dimensionless interval function $B^{I}(x) B^{I}(\xi)$ which is related to the midpoint of the interval function $E^{* I}(x) E^{* I}(\xi)$ as specified in Eq. (11).

If the mid $\{\cdot\}$ operator is viewed as the analogue of the stochastic average operator, within the interval framework, the function $\Gamma_{B}(x, \xi)$ in Eq. (11) may be regarded as the non-probabilistic counterpart of the autocorrelation function characterizing probabilistically a random field. Based on this analogy, a proper generalization of the Karhunen-Loève decomposition is applied here, i.e.:
$\Gamma_{B}(x, \xi)=\sum_{i=1}^{\infty} \lambda_{i} \psi_{i}(x) \psi_{i}(\xi) \Rightarrow \Gamma_{B}(x, x) \equiv \operatorname{mid}\left\{\left(B^{I}(x)\right)^{2}\right\}=\sum_{i=1}^{\infty} \lambda_{i} \psi_{i}^{2}(x)$

where $\lambda_{i},(i=1,2, \ldots)$, is the ith eigenvalue of the bounded symmetric non-negative function, $\Gamma_{B}(x, \xi)$, and $\psi_{i}(x)$ is the corresponding eigenfunction, which satisfies the following orthogonality condition:

$\int_{0}^{L} \psi_{i}(x) \psi_{j}(x) d x= \begin{cases}1 & \text { if } i=j \\ 0 & \text { if } i \neq j .\end{cases}$

The eigenproperties of the function $\Gamma_{B}(x, \xi)$ are found by solving the following homogeneous Fredholm integral equation of the second kind:

$\int_{0}^{L} \Gamma_{B}(x, \xi) \psi_{i}(x) d x=\lambda_{i} \psi_{i}(\xi)$.

The eigenvalues solutions of this eigenproblem are real positive numbers and the associated eigenfunctions are real functions. Notice that the expansion in Eq. (12) is usually truncated after $N$ terms to reduce the computational burden of the subsequent structural analysis.

Based on the decomposition (12) and taking into account Eq. (11), the following expression of the dimensionless interval function $B^{I}(x)$ is readily found:

$B^{I}(x)=\sum_{i=1}^{N} \sqrt{\lambda_{i}} \psi_{i}(x) \hat{e}_{i}^{I}, \quad x \in[0, L]$.

It can be observed that Eq. (12) allows to express the dimensionless interval field $B^{I}(x)$ as superposition of $N$ interval functions associated to the EUI variables $\hat{e}_{i}^{I}(i=1,2, \ldots, N)$.

Upon substitution of Eq. (15) into Eq. (8), the interval function $E^{* I}(x)$ can be rewritten in the following form:

$E^{* I}(x)=E_{0}^{*}\left[1+\sum_{i=1}^{N} \sqrt{\lambda_{i}} \psi_{i}(x) \hat{e}_{i}^{I}\right], \quad x \in[0, L]$

where $\hat{e}_{i}^{I}$ is the $i$ th EUI variable.

Then, the lower bound and upper bound, $E^{*}(x)$ and $\bar{E}^{*}(x)$, of the interval Young's modulus $E^{* I}(x)$ in Eq. (16) can be defined as:

$\underline{E}^{*}(x)=E_{0}^{*}[1-\Delta B(x)] ; \quad \bar{E}^{*}(x)=E_{0}^{*}[1+\Delta B(x)], \quad x \in[0, L]$

with

$\Delta B(x)=\frac{\Delta E^{*}(x)}{E_{0}^{*}}=\sum_{i=1}^{N}\left|\sqrt{\lambda_{i}} \psi_{i}(x)\right|, \quad x \in[0, L]$

where $|\cdot|$ denotes the absolute value of .

It is worth emphasizing that the proposed definition of the interval field (16) is formally analogous to the model proposed by Verhaeghe et al. [17]. The main difference consists in the use of the EUI variable $\hat{e}_{i}^{I}$ and of the basic features of the improved interval analysis [15], briefly summarized in the previous subsection. Indeed, it can be readily verified that substituting Eq. (15) into Eq. (11) and taking into account the properties of the EUI variable reported in Eq. (4), the function $\Gamma_{B}(x, \xi)$ defined in Eq. (12) is recovered. This result cannot be obtained by means of the "ordinary" interval analysis (see Appendix B).

The consistency of the presented interval field definition can also be assessed by examining the two limit cases of total spatial dependency and spatially independency of the uncertain property. To this aim, let us assume that the function governing the spatial dependency of the interval field $E^{* I}(x)$ has the following exponential form:

$\Gamma_{B}(x, \xi)=C_{B}^{2} \exp \left(-\frac{|x-\xi|}{l_{B}}\right)$ 
where $C_{B}$ and $l_{B}$ are appropriate parameters. It can be argued that $C_{B}$ affects the deviation amplitude of the interval field, while the value of $l_{B}$ actually rules the spatial dependency of the uncertain property. In other words, $l_{B}$ may be regarded as the non-probabilistic counterpart of the correlation length characterizing random fields. In fact, as $l_{B}$ decreases only values of the Young's modulus at close locations are dependent on each other. On the other hand, if $l_{B} \rightarrow \infty$, the function (19) reduces to $C_{B}^{2}$. Physically, this circumstance corresponds to the total dependency condition in which the uncertain property is described by a single interval variable constant over the whole domain $[0, L]$, that is the symmetric interval function $B^{I}(x)$ reduces to:

$B^{I}(x) \equiv b^{I}=b \hat{e}^{I}$

The radius $b$ of the symmetric interval variable $b^{I}$ in Eq. (20) can be evaluated from Eq. (11) which yields:

$\operatorname{mid}\left\{B^{I}(x) B^{I}(\xi)\right\}=b^{2}=C_{B}^{2} \Rightarrow b=C_{B}$

being $\hat{e}^{I} \times \hat{e}^{I}=[1,1]$ (see Eq. (4)) and $\Gamma_{B}(x, \xi) \rightarrow C_{B}^{2}$. Then, the interval Young's modulus is defined as follows:

$E^{* I}=E_{0}^{*}\left(1+b e^{I}\right)$

where $b=C_{B}$. The bounds of $E^{* I}$ read:

$\underline{E}^{*}=E_{0}^{*}(1-b) ; \quad \bar{E}^{*}=E_{0}^{*}(1+b)$.

On the other extreme, if $l_{B} \rightarrow 0$ the uncertain property turns out to be spatially independent and the proposed interval field model reduces to a series of independent interval variables, one for each grid point or element of the discretized domain $[0, L]$.

\section{Long-range interactions in presence of uncertain-but- bounded elastic modulus}

\subsection{Interval integro-differential equilibrium equation}

The equation governing the response of the 1D heterogeneous solid with uncertain-but-bounded elastic modulus in presence of long-range interactions can be readily derived by substituting the expression (16) of the interval elastic modulus $E^{* I}(x)$ into Eq. (2), which yields the following interval integro-differential equation:

$$
\begin{aligned}
& E_{0}^{*} \frac{d}{d x}\left[A(x) \frac{d u^{I}(x)}{d x}\right]+E_{0}^{*} \sum_{i=1}^{N} \sqrt{\lambda_{i}} \hat{e}_{i}^{I} \frac{d}{d x}\left[A(x) \psi_{i}(x) \frac{d u^{I}(x)}{d x}\right] \\
& \quad+c_{q} A(x) \rho^{2} \int_{0}^{L} A(\xi) g(x, \xi)\left[u^{I}(\xi)-u^{I}(x)\right] d \xi \\
& \quad=-A(x) b(x)
\end{aligned}
$$

where $u^{I}(x)$ denotes the interval displacement function. Equation (24) must be supplemented by the pertinent kinematic and static boundary conditions given by Eq. (3), with the interval Young's modulus defined as in Eq. (16).

Following the strategy commonly adopted within of a nonlocal deterministic setting [27], a finite difference discretization of Eq. (24) is performed here by subdividing the bar domain $[0, L]$ into $n$ intervals of amplitude $\Delta x$, so that $x_{j}=(j-1) \Delta x$ denotes the abscissa of the $j$-th grid point with $j=1,2, \ldots, n$. After multiplying both sides by $\Delta x$, the discretized form of Eq. (24) reads:

$$
\begin{aligned}
& \frac{E_{0}^{*}}{\Delta x}\left[A_{j} u_{j+1}^{I}-\left(A_{j}+A_{j-1}\right) u_{j}^{I}+A_{j-1} u_{j-1}^{I}\right] \\
& \quad+\sum_{i=1}^{N}\left[s_{i j} u_{j+1}^{I}-\left(s_{i j}+s_{i j-1}\right) u_{j}^{I}+s_{i j-1} u_{j-1}^{I}\right] \hat{e}_{i}^{I} \\
& \quad+c_{q}(\rho \Delta x)^{2} A_{j} \sum_{r=1}^{n} A_{r} g\left(x_{j}, x_{r}\right)\left(u_{r}^{I}-u_{j}^{I}\right)=-b_{j} A_{j} \Delta x
\end{aligned}
$$

where $A_{j}=A\left(x_{j}\right), u_{j}^{I}=u^{I}\left(x_{j}\right), b_{j}=b\left(x_{j}\right)$ and $s_{i j}=\left(E_{0}^{*} \psi_{i j} A_{j} \sqrt{\lambda_{i}}\right) / \Delta x$, with $\psi_{i j}=\psi_{i}\left(x_{j}\right),(i=1,2, \ldots, N ; j=1,2, \ldots, n)$.

Introducing the following positions:

$k_{j}^{*}=\frac{E_{0}^{*} A_{j}}{\Delta x} ; \quad k_{j r}^{(n l)}=c_{q} A_{j} A_{r}(\rho \Delta x)^{2} g\left(x_{j}, x_{r}\right)$,

the set of linear interval equations in Eq. (25) can be written in compact form as:

$\mathbf{K}^{I} \mathbf{u}^{I}=\left(\mathbf{K}_{0}+\Delta \mathbf{K}_{B}^{I}\right) \mathbf{u}^{I}=\mathbf{F}$

where $\mathbf{u}^{I}$ is the vector of order $n$ collecting the interval displacements $u_{j}^{I}$ at the grid points $x_{j},(j=1,2, \ldots, n) ; \mathbf{F}$ is a $n$-vector whose $j$-th element, $F_{j}=b_{j} A_{j} \Delta x$, is the resultant of the body force field applied at the grid point $x_{j} ; \mathbf{K}_{0}=\mathbf{K}^{*}+\mathbf{K}^{(n l)}$ is the stiffness matrix of the nominal system, i.e. with $E^{*}(x)=E_{0}^{*}$, which can be evaluated as the sum of the local and non-local stiffness matrices, $\mathbf{K}^{*}$ and $\mathbf{K}^{(n l)}$, respectively, defined as:

$$
\begin{gathered}
\mathbf{K}^{*}=\left[\begin{array}{cccccc}
k_{1}^{*} & -k_{1}^{*} & 0 & 0 & \ldots & 0 \\
-k_{1}^{*} & k_{1}^{*}+k_{2}^{*} & -k_{2}^{*} & 0 & \ldots & 0 \\
0 & -k_{2}^{*} & k_{2}^{*}+k_{3}^{*} & -k_{3}^{*} & \ldots & 0 \\
\vdots & \vdots & \vdots & \vdots & \ddots & \vdots \\
0 & 0 & 0 & 0 & \ldots & k_{n-1}^{*}+k_{n}^{*}
\end{array}\right] ; \\
\mathbf{K}^{(n l)}=\left[\begin{array}{ccccc}
k_{11}^{(n l)} & -k_{12}^{(n l)} & -k_{13}^{(n l)} & \ldots & -k_{1 n}^{(n l)} \\
\ldots & k_{22}^{(n l)} & -k_{23}^{(n l)} & \ldots & -k_{2 n}^{(n l)} \\
\ldots & \ldots & \ldots & \ldots & \ldots \\
\ldots & S Y M & \ldots & \ldots & -k_{n-1 n}^{(n l)} \\
\ldots & \cdots & \ldots & \ldots & k_{n n}^{(n l)}
\end{array}\right]
\end{gathered}
$$

The diagonal terms of the matrix $\mathbf{K}^{(n l)}$ read $k_{j j}^{(n l)}=\sum_{\substack{r=1 \\ r \neq j}}^{n} k_{j r}^{(n l)}$. Notice that in Eq. (28b), the main features of the distance-decaying function, $g(x, \xi)$, are fulfilled and the matrices $\mathbf{K}^{*}$ and $\mathbf{K}^{(n l)}$ turn out to be symmetric and positive-definite matrices also for heterogeneous materials.

The interval stiffness matrix $\mathbf{K}^{I}$ in Eq. (27) involves an additional local contribution given by the interval matrix $\Delta \mathbf{K}_{B}^{I}$ associated to the EUI variables, which can be expressed as:

$\Delta \mathbf{K}_{B}^{I}=\sum_{i=1}^{N} \Delta \mathbf{S}_{B, i} \hat{e}_{i}^{I}$.

In the previous equation, $\Delta \mathbf{S}_{B, i}$ is a tridiagonal symmetric and positive-definite matrix, given by:

$$
\Delta \mathbf{S}_{B, i}=\left[\begin{array}{cccccc}
s_{i 1} & -s_{i 1} & 0 & 0 & \cdots & 0 \\
-s_{i 1} & s_{i 1}+s_{i 2} & -s_{i 2} & 0 & \cdots & 0 \\
0 & -s_{i 2} & s_{i 2}+s_{i 3} & -s_{i 3} & \cdots & 0 \\
\vdots & \vdots & \vdots & \vdots & \ddots & \vdots \\
0 & 0 & 0 & 0 & \cdots & s_{i n-1}+s_{i n}
\end{array}\right] .
$$

The finite difference discretization of the integro-differential Eq. (24) shows that the discrete counterpart of the continuous mechanically-based model is equivalent to a point-spring network [27]. 
Within a deterministic setting (see Eq. (2)), indeed the contact forces are represented by linear springs of axial stiffness $k_{j}^{*}$ (see Eq. (26a)) and the long-range interactions are described by linear springs of distance-decaying stiffness $k_{j r}^{(n l)}$ (see Eq. (26b)) connecting all non-adjacent points. When the Young's modulus is modelled as an interval field according to Eq. (16), additional contributions to the contact forces arise (see Eq. (25)) which are mechanically equivalent to linear springs of interval axial stiffness.

\subsection{Bounds of the interval response}

Once the finite difference discretization of Eq. (24) has been performed, the solution of the problem consists in the evaluation of the narrowest interval $\mathbf{u}^{I}$ containing all possible vectors, $\mathbf{u}$, satisfying Eq. (27), when the elements of the matrix $\Delta \mathbf{S}_{B, i} \hat{e}_{i}^{I}$ assume all possible values inside the intervals $\left[-s_{i j},+s_{i j}\right]$. Preliminarily, it is useful to underline that the square interval matrix $\mathbf{K}^{I}$ is regular, that is each matrix $\mathbf{K} \in \mathbf{K}^{I}$ is non-singular [37] (see Appendix B). Therefore, the solution of Eq. (27) exists for all $\mathbf{K} \in \mathbf{K}^{I}$ and can be written, by adopting the interval formalism, as:

$\mathbf{u}^{I}=\left(\mathbf{K}_{0}+\Delta \mathbf{K}_{B}^{I}\right)^{-1} \mathbf{F}=\left(\mathbf{K}_{0}+\sum_{i=1}^{N} \Delta \mathbf{S}_{B, i} \hat{e}_{i}^{I}\right)^{-1} \mathbf{F}$.

Under the assumption of small dimensionless deviation of the interval elastic modulus, i.e. $\Delta B(x) \ll 1$ for all $x \in[0, L]$, an efficient procedure for the solution of the set of linear interval equations (27) is herein proposed. The procedure relies on the following decomposition of the local interval matrix $\Delta \mathbf{K}_{B}^{I}$ :

$\Delta \mathbf{K}_{B}^{I}=\sum_{i=1}^{N} \Delta \mathbf{S}_{B, i} \hat{e}_{i}^{I}=\sum_{i=1}^{N} \sum_{\ell=1}^{n} \mathbf{s}_{B, i} \mathbf{w}_{\ell}^{T} \hat{e}_{i}^{I}$

where $\mathbf{s}_{B, i \ell}$ is the $\ell$ th column of the matrix $\Delta \mathbf{S}_{B, i}$ in Eq. (30) and $\mathbf{w}_{\ell}$ is a column vector of order $n$ containing all zeros except the $\ell$ th element which is equal to 1 . Substituting Eq. (32) into Eq. (27), the interval stiffness matrix $\mathbf{K}^{I}$ takes the following form:

$\mathbf{K}^{I}=\mathbf{K}_{0}+\Delta \mathbf{K}_{B}^{I}=\mathbf{K}_{0}+\sum_{i=1}^{N} \sum_{\ell=1}^{n} \mathbf{s}_{B, i} \mathbf{w}_{\ell}^{T} \hat{e}_{i}^{I}$.

Notice that, by virtue of the decomposition (32), the deviation, $\Delta \mathbf{K}_{B}^{I}$, with respect to the nominal stiffness matrix, $\mathbf{K}_{0}$, is expressed as superposition of $N \times n$ modifications of rank one. Then, following the formulation proposed by Muscolino and Sofi [38,39], after some algebra, the approximate inverse of the interval stiffness matrix in Eq. (33) can be evaluated in explicit form as:

$\left(\mathbf{K}_{0}+\Delta \mathbf{K}_{B}^{I}\right)^{-1} \approx \mathbf{K}_{0}^{-1}-\sum_{i=1}^{N} \sum_{\ell=1}^{n} \frac{\hat{e}_{i}^{I}}{1+\hat{e}_{i}^{I} d_{B, i \ell}} \mathbf{D}_{B, i \ell}$

where the following quantities have been introduced:

$d_{B, i \ell}=\left|\mathbf{w}_{\ell}^{T} \mathbf{K}_{0}^{-1} \mathbf{s}_{B, i \ell}\right| ; \mathbf{D}_{B, i \ell}=\mathbf{K}_{0}^{-1} \mathbf{s}_{B, i \ell} \mathbf{w}_{\ell}^{T} \mathbf{K}_{0}^{-1}$.

Eq. (34) holds if and only if the following condition is satisfied:

$d_{B, i \ell}<1$.

Upon rewriting the ratio appearing in the summation in Eq. (34) in affine form, the following approximate explicit expression of the interval vector solution $\mathbf{u}^{I} \in \mathbb{Q} \mathbb{R}^{n}$ is obtained:

$\mathbf{u}^{I} \approx\left[\mathbf{K}_{0}^{-1}+\sum_{i=1}^{N} \sum_{\ell=1}^{n}\left(a_{0, i \ell}+\Delta a_{i \ell} \hat{e}_{i}^{I}\right) \mathbf{D}_{B, i \ell}\right] \mathbf{F}$

where the quantities $a_{0, i \ell}$ and $\Delta a_{i \ell}$, after some interval algebra, can be written as: $a_{0, i \ell}=\frac{d_{B, i \ell}}{1-d_{B, i \ell}^{2}} ; \quad \Delta a_{i \ell}=\frac{1}{1-d_{B, i \ell}^{2}}$.

From an engineering point of view, within the interval framework, the main goal of structural analysis is the evaluation of the narrowest interval which certainly contains the response. This interval is bounded by the lower and upper bounds, $\underline{\mathbf{u}}$ and $\overline{\mathbf{u}}$, of the interval response vector $\mathbf{u}^{I}$. Based on the explicit solution in Eq. (37) and adopting the interval formalism, the vectors $\underline{\mathbf{u}}$ and $\overline{\mathbf{u}}$ can be evaluated as follows:

$\underline{\mathbf{u}}=\mathbf{u}_{0}-\Delta \mathbf{u} ; \overline{\mathbf{u}}=\mathbf{u}_{0}+\Delta \mathbf{u}$,

where

$\mathbf{u}_{0}=\left(\mathbf{K}_{0}^{-1}+\sum_{i=1}^{N} \sum_{\ell=1}^{n} a_{0, i \ell} \mathbf{D}_{B, i \ell}\right) \mathbf{F} ; \quad \Delta \mathbf{u}=\sum_{i=1}^{N}\left|\sum_{\ell=1}^{n} \Delta a_{i \ell} \mathbf{D}_{B, i \ell} \mathbf{F}\right|$

are the midpoint and the deviation of the interval displacement vector $\mathbf{u}^{I}$. The symbol $|\cdot|$ in Eq. (40b) denotes the component wise absolute value.

\section{Long-range interactions in presence of randomly varying elastic modulus}

\subsection{Random field}

Following the well-known probabilistic approach, the Young's modulus function $\tilde{E}^{*}(x)$ of the $1 \mathrm{D}$ heterogeneous solid with longrange interactions is now modelled as a homogeneous Gaussian random field, defined as:

$\tilde{E}^{*}(x)=E_{0}^{*}[1+\tilde{B}(x)], \quad x \in[0, L]$

where $\tilde{B}(x)$ is a homogeneous zero-mean Gaussian random field describing the dimensionless fluctuation of the elastic modulus about the nominal or mean-value $E_{0}^{*}$. The random field $\tilde{B}(x)$ must satisfy the mathematical restriction $|\tilde{B}(x)|<1$ to yield always positive values of $\tilde{E}^{*}(x)$. Notice that, such a condition is not mathematically satisfied for Gaussian random fields, but as small fluctuations are considered in the analysis, then we may assume a Gaussian distribution of the elastic modulus along the bar axis. Let $\mathrm{E}\langle\cdot\rangle$ denote the mathematical expectation operator so that first-and second-order statistics of the random field $\tilde{B}(x)$ read:

$\mu_{\tilde{B}}(x)=\mathrm{E}\langle\tilde{B}(x)\rangle=0 ; \quad R_{\tilde{B} \tilde{B}}(x, \xi)=\mathrm{E}\langle\tilde{B}(x) \tilde{B}(\xi)\rangle$.

By applying the Karhunen-Loève decomposition, the autocorrelation function $R_{\tilde{B} \tilde{B}}(x, \xi)$ of the random field $\tilde{B}(x)$ can be expressed as:

$R_{\tilde{B} \tilde{B}}(x, \xi)=\sum_{i=1}^{\infty} \tilde{\lambda}_{i} \tilde{\psi}_{i}(x) \tilde{\psi}_{i}(\xi) \Rightarrow \sigma_{\tilde{B}}^{2}(x) \equiv R_{\tilde{B} \tilde{B}}(x, x)=\sum_{i=1}^{\infty} \tilde{\lambda}_{i} \tilde{\psi}_{i}^{2}(x)$,

where $\tilde{\lambda}_{i}(i=1,2, \ldots)$ is the $i$-th eigenvalue of the bounded symmetric non-negative function $R_{\tilde{B} \tilde{B}}(x, \xi)$ and $\tilde{\psi}_{i}(x)$ denotes the corresponding eigenfunction, which satisfies the orthogonality condition (13). The eigenproperties of the autocorrelation function $R_{\tilde{B} \tilde{B}}(x, \xi)$ can be evaluated by solving the following integral eigenproblem:

$\int_{0}^{L} R_{\tilde{B} \tilde{B}}(x, \xi) \tilde{\psi}_{i}(x) d x=\tilde{\lambda}_{i} \tilde{\psi}_{i}(\xi)$.

The eigenvalues are real positive numbers and the associated eigenfunctions are real functions. As usual, the expansion (43) is truncated after $M$ terms to reduce the computational effort of the subsequent stochastic structural analysis. Then, the random field $\tilde{B}(x)$ can be expressed as summation of deterministic functions combined with a set of uncorrelated Gaussian zero-mean random variables $\tilde{Z}_{i}$ : 
$\tilde{B}(x)=\sum_{i=1}^{M} \sqrt{\tilde{\lambda}_{i}} \tilde{\psi}_{i}(x) \tilde{Z}_{i}$

with

$$
\mathrm{E}\left\langle\tilde{Z}_{i}\right\rangle=0, \forall i ; \quad \mathrm{E}\left\langle\tilde{Z}_{i} \tilde{Z}_{j}\right\rangle= \begin{cases}1 & \text { if } i=j \\ 0 & \text { if } i \neq j .\end{cases}
$$

\subsection{Stochastic integro-differential equilibrium equation}

Substituting Eq. (45) into Eq. (41) and then the resulting expression of the randomly varying elastic modulus $\tilde{E}^{*}(x)$ into Eq. (2), the following stochastic integro-differential equation is obtained:

$$
\begin{aligned}
E_{0}^{*} & \frac{d}{d x}\left[A(x) \frac{d U(x)}{d x}\right]+E_{0}^{*} \sum_{i=1}^{M} \sqrt{\tilde{\lambda}_{i}} \tilde{Z}_{i} \frac{d}{d x}\left[A(x) \tilde{\psi}_{i}(x) \frac{d U(x)}{d x}\right] \\
& +c_{q} A(x) \rho^{2} \int_{0}^{L} A(\xi) g(x, \xi)[U(\xi)-U(x)] d \xi \\
& =-A(x) b(x)
\end{aligned}
$$

where the capital letter $U(x)$ denotes the random displacement field. Eq. (47) must be supplemented by appropriate kinematic and static boundary conditions given by Eqs. $(3 a, b)$ with the randomly varying Young's modulus.

The solution of Eq. (47) can be obtained resorting to the finite difference method discussed in the previous section (see also [40]). To this aim, let us introduce a discretization grid of the domain into intervals of amplitudes $\Delta x$ so that, after multiplying both sides by $\Delta x$, Eq. (47) takes the following discretized form:

$$
\begin{aligned}
& \frac{E_{0}^{*} \Delta x}{\Delta x}\left[A_{j} U_{j+1}-\left(A_{j}+A_{j-1}\right) U_{j}+A_{j-1} U_{j-1}\right] \\
& \quad+\sum_{i=1}^{M}\left[\tilde{s}_{i j} U_{j+1}-\left(\tilde{s}_{i j}+\tilde{s}_{i j-1}\right) U_{j}+\tilde{s}_{i j-1} U_{j-1}\right] \tilde{Z}_{i} \\
& \quad+c_{q}(\rho \Delta x)^{2} A_{j} \sum_{r=1}^{n} A_{r} g\left(x_{j}, x_{r}\right)\left(U_{r}-U_{j}\right) \\
& \quad=-b_{j} A_{j} \Delta x
\end{aligned}
$$

where $\quad U_{j}=U\left(x_{j}\right) \quad$ and $\quad \tilde{s}_{i j}=\left(E_{0}^{*} \tilde{\psi}_{i j} A_{j} \sqrt{\tilde{\lambda}_{i}}\right) / \Delta x \quad$ with $\tilde{\psi}_{i j}=\tilde{\psi}_{i}\left(x_{j}\right),(i=1,2, \ldots, M ; j=1,2, \ldots, n)$. The set of linear algebraic equations with random coefficients in Eq. (48) can be written in compact form as:

$$
\tilde{\mathbf{K}} \mathbf{U}=\left(\mathbf{K}_{0}+\Delta \tilde{\mathbf{K}}_{\tilde{B}}\right) \mathbf{U}=\mathbf{F}
$$

where $\mathbf{U}$ is the vector of order $n$ collecting the stochastic displacements $U_{j}(j=1,2, \ldots n)$ at the grid points $x_{j} ; \mathbf{K}_{0}=\mathbf{K}^{*}+\mathbf{K}^{(n l)}$ is the stiffness matrix of the nominal system (see Eqs. (28a,b)). Notice that the stiffness matrix $\tilde{\mathbf{K}}$ in Eq. (49) has a stochastic nature due to the contribution of the random matrix $\Delta \tilde{\mathbf{K}}_{\tilde{B}}$ associated to the uncorrelated random variables $\tilde{Z}_{i}$, whereas the vector $\mathbf{F}$ is deterministic and coincides with the one defined in the previous section (see Eq. (27)). Finally, it is observed that the additional stochastic stiffness matrix $\Delta \tilde{\mathbf{K}}_{\tilde{B}}$ can be written as:

$\Delta \tilde{\mathbf{K}}_{\tilde{B}}=\sum_{i=1}^{M} \Delta \tilde{\mathbf{S}}_{\tilde{B}, i} \tilde{Z}_{i}$

where $\Delta \tilde{\mathbf{S}}_{\tilde{B}, i}$ is a tridiagonal symmetric and positive-definite matrix given by:

$$
\Delta \tilde{\mathbf{S}}_{\tilde{B}, i}=\left[\begin{array}{cccccc}
\tilde{s}_{i 1} & -\tilde{s}_{i 1} & 0 & 0 & \ldots & 0 \\
-\tilde{s}_{i 1} & \tilde{s}_{i 1}+\tilde{s}_{i 2} & -\tilde{s}_{i 2} & 0 & \ldots & 0 \\
0 & -\tilde{s}_{i 2} & \tilde{s}_{i 2}+\tilde{s}_{i 3} & -\tilde{s}_{i 3} & \ldots & 0 \\
\vdots & \vdots & \vdots & \vdots & \ddots & \vdots \\
0 & 0 & 0 & 0 & \ldots & \tilde{s}_{i n-1}+\tilde{s}_{i n}
\end{array}\right]
$$

which is formally analogous to the matrix defined in Eq. (30).
It is worth mentioning that the effects of random material properties on the response of a 1D non-local elastic solid have been recently analyzed in the context of the mechanically-based approach by modeling the mass density as a homogeneous Gaussian stochastic field [32]. Under this assumption, the long-range interactions are affected by the material randomness because they are taken as depending on the elementary interacting masses. Conversely, the problem addressed in the present study always involves deterministic long-range forces since the uncertain Young's modulus affects only the local contact forces.

Finally, it is noted that the probabilistic model of the uncertain Young's modulus leads to a mechanically equivalent point-spring network where linear springs of random axial stiffness describe the additional stochastic contribution to the contact forces.

\subsection{Mean-value and covariance of the stochastic response}

As known, the solution of Eq. (49) depends on the realization of the random field $\tilde{B}(x)$ describing the fluctuation of the elastic modulus along the 1D solid. Therefore, direct inversion of the random stiffness matrix $\tilde{\mathbf{K}}$, providing the displacement vector $\mathbf{U}=\tilde{\mathbf{K}}^{-1} \mathbf{F}$, is not useful to evaluate the statistics of the mechanical response of the bar unless the onerous Monte Carlo Simulation (MCS) method is used.

In order to avoid MCS, the stochastic matrix $\Delta \tilde{\mathbf{K}}_{\tilde{B}}$ in Eq. (50) can be decomposed according to Eq. (32), as:

$\Delta \tilde{\mathbf{K}}_{\tilde{B}}=\sum_{i=1}^{M} \Delta \tilde{\mathbf{S}}_{\tilde{B}, i} \tilde{Z}_{i}=\sum_{i=1}^{M} \sum_{\ell=1}^{n} \tilde{\mathbf{S}}_{\tilde{B}, i \ell} \mathbf{w}_{\ell}^{T} \tilde{Z}_{i}$

where $\tilde{\mathbf{s}}_{\tilde{B}, i \ell}$ is the $\ell$ th column of the matrix $\Delta \tilde{\mathbf{S}}_{\tilde{B}, i}$ (see Eq. (51)), whereas $\mathbf{w}_{\ell}$ is a column vector of order $n$ containing all zeros except the $\ell$ th element which is equal to 1 . Based on Eq. (52), the approximate inverse of the matrix $\tilde{\mathbf{K}}$ can be evaluated by applying an expression analogous to the one given in Eq. (34). Accordingly, the solution of the set of linear stochastic equations (49) can be written in the following approximate explicit form:

$\mathbf{U}=\left(\mathbf{K}_{0}+\Delta \tilde{\mathbf{K}}_{\tilde{B}}\right)^{-1} \mathbf{F} \approx \mathbf{K}_{0}^{-1} \mathbf{F}-\sum_{i=1}^{M} \sum_{\ell=1}^{n} \frac{\tilde{Z}_{i}}{1+\tilde{d}_{\tilde{B}, i \ell} \tilde{Z}_{i}} \tilde{\mathbf{D}}_{\tilde{B}, i \ell} \mathbf{F}$

where $\mathbf{K}_{0}$ is the stiffness matrix of the nominal system and

$\tilde{d}_{\tilde{B}, i \ell}=\left|\mathbf{w}_{\ell}^{T} \mathbf{K}_{0}^{-1} \tilde{\mathbf{s}}_{\tilde{B}, i \ell}\right| ; \quad \tilde{\mathbf{D}}_{\tilde{B}, i \ell}=\mathbf{K}_{0}^{-1} \tilde{\mathbf{s}}_{\tilde{B}, i \ell} \mathbf{W}_{\ell}^{T} \mathbf{K}_{0}^{-1}$.

Notice that Eq. (53) holds provided that the following condition is satisfied:

$\tilde{d}_{\tilde{B}, i \ell}<1$.

Finally, the mean-value vector and the covariance matrix of the stochastic response vector $\mathbf{U}$ can be evaluated, respectively, as follows:

$$
\begin{aligned}
\boldsymbol{\mu}_{\mathbf{U}}= & \mathrm{E}\langle\mathbf{U}\rangle \approx \mathbf{K}_{0}^{-1} \mathbf{F}-\sum_{i=1}^{M} \sum_{\ell=1}^{n} \mathrm{E}\left\langle\chi_{i \ell}\right\rangle \tilde{\mathbf{D}}_{\tilde{B}, i \ell} \mathbf{F} ; \\
\boldsymbol{\Sigma}_{\mathbf{U}}= & \mathrm{E}\left\langle\mathbf{U} \mathbf{U}^{T}\right\rangle-\boldsymbol{\mu}_{\mathbf{U}} \boldsymbol{\mu}_{\mathbf{U}}^{T} \approx \sum_{i=1}^{M} \sum_{\ell=1}^{n} \sum_{m=1}^{n}\left[\mathrm{E}\left\langle\chi_{i \ell} \chi_{i m}\right\rangle\right. \\
& \left.-\mathrm{E}\left\langle\chi_{i \ell}\right\rangle \mathrm{E}\left\langle\chi_{i m}\right\rangle\right] \tilde{\mathbf{D}}_{\tilde{B}, i \ell} \mathbf{F F}^{T} \tilde{\mathbf{D}}_{\tilde{B}, i m}^{T},
\end{aligned}
$$

where:

$\chi_{i k}=\frac{\tilde{Z}_{i}}{1+\tilde{d}_{\tilde{B}, i k} \tilde{Z}_{i}},(k=\ell, m)$.

The previous equations provide substantial computational savings over classical MCS method since they just involve the statistics of 
the random variables $\chi_{i k}$ (see Eq. (57)) without requiring the inversion of the global stochastic stiffness matrix. Furthermore, the closed-form expression of the random response in Eq. (53) enables one to evaluate higher-order statistical moments useful to determine the probability density function of the response.

In particular, based on Eq. (53) and taking into account that the random variables $\tilde{Z}_{i}$ are uncorrelated, the $\mu$ th order statistical moment of the $v$ th nodal displacement can be formally evaluated as:
$c_{q}=\frac{c}{\rho^{2}}=\frac{\mathrm{E}_{0}\left(1-\beta_{1}\right)}{2 A^{2} l_{0} \rho^{2}}$.

Due to the lack of rigorous values from experimental tests, the parameters $l_{0}$ and $\beta_{1}$, entering the non-local terms, have been set so as to enhance non-local effects, say $l_{0}=5 \mathrm{~nm}$ and $\beta_{1}=0.7$.

The analysis of the axial displacement field in the CNT has been performed resorting to both interval and stochastic modeling of

$$
\begin{aligned}
& \mathrm{E}\left\langle U_{\tilde{B}, v}^{\mu}\right\rangle=u_{0, v}^{\mu}-\mu u_{0, v}^{\mu-1} \sum_{i=1}^{M} \sum_{p=1}^{n} m_{\tilde{B}, i p}^{(1)} g_{v, i p}+\frac{\mu(\mu-1)}{2} u_{0, v}^{\mu-2}\left[\sum_{p=1}^{n} \sum_{q=1}^{n} \sum_{i=1}^{M} m_{\tilde{B}, i p q}^{(2)} g_{v, i p} g_{v, i q}+\sum_{i=1}^{M} \sum_{\substack{p=1 \\
j}}^{n} \sum_{\substack{j=1 \\
j \neq i}}^{M} \sum_{q=1}^{n} m_{\tilde{B}, i p}^{(1)} m_{\tilde{B}, j q}^{(1)} g_{v, i p} g_{v, j q}\right]+\cdots \\
& +(-1)^{\mu}\left[\sum_{i=1}^{M} \sum_{p=1}^{n} \sum_{q=1}^{n} \sum_{r=1}^{n} \cdots m_{\tilde{B}, i p q r . \ldots}^{(\mu)} g_{v, i p} g_{v, i q} g_{v, i r} \cdots+\sum_{i=1}^{M} \sum_{\substack{j=1 \\
j \neq i}}^{M} \sum_{p=1}^{n} \sum_{q=1}^{n} \sum_{r=1}^{n} \cdots m_{\tilde{B}, i p}^{(1)} m_{\tilde{B}, j q r \cdots}^{(\mu-1)} g_{v, i p} g_{v, j q} g_{v, j r} \cdots+\cdots\right]
\end{aligned}
$$

where $u_{0, v}^{\rho}$ is the $\rho$ th power $(\rho=1,2, \ldots, \mu)$ of the $v$ th element of the vector $\mathbf{u}_{0}=\mathbf{K}_{0}^{-1} \mathbf{F} ; g_{v, \ell p}$ is the $v$ th element $(\ell=i, j, k \ldots)$ of the vector $\left\{\tilde{\mathbf{D}}_{\tilde{B}, \ell p} \mathbf{F}\right\} ; m_{\tilde{B}, \ell p q r . \ldots}^{(\rho)}$ denotes the $\rho-$ th statistical moment of the random variables (57) which is defined as follows: the uncertain Young's modulus $E^{*}(x)$. For comparison purposes, the deterministic symmetric non-negative bounded function $\Gamma_{B}(x, \xi)$, governing the spatial dependency between adjacent values of the dimensionless interval function $B^{I}(x)$ in Eq. (8), has been

$m_{\tilde{B}, \ell p q r \ldots}^{(\rho)}=\int_{-\infty}^{+\infty}\left(\frac{\tilde{z}_{\ell}}{1+\tilde{d}_{\tilde{B}, \ell p} \tilde{z}_{\ell}}\right)\left(\frac{\tilde{z}_{\ell}}{1+\tilde{d}_{\tilde{B}, \ell q} \tilde{z}_{\ell}}\right)\left(\frac{\tilde{z}_{\ell}}{1+\tilde{d}_{\tilde{B}, \ell r} \tilde{z}_{\ell}}\right) \cdots(\rho$ time $) \cdots p_{\tilde{z}_{\ell}}\left(\tilde{z}_{\ell}\right) \mathrm{d} \tilde{z}_{\ell} ; \quad \rho=1, \ldots, \mu \quad \ell=i, j, k \ldots$

The previous expression takes into account that the zero-mean random variables $\tilde{Z}_{\ell}$ posses one-dimensional Gaussian probability density function $p_{\tilde{z}_{\ell}}\left(\tilde{z}_{\ell}\right)$.

As a final remark, it is noted that by using the Karhunen-Loève decomposition in conjunction with the finite difference method, a set of linear stochastic equations (see Eq. (49)) formally analogous to the one governing the displacement field (see Eq. (27)) in the context of the interval model is obtained. This analogy allowed us to apply the same approach for deriving approximate closedform expressions of the statistics and bounds of the response.

\section{Numerical application}

For validation purposes, the response of a carbon nanotube (CNT) with uncertain Young's modulus, fixed at $x=0$ and subjected to a tensile force $F=1 \mathrm{nN}$ at the free end, $x=L$, has been analyzed. The geometrical and mechanical properties have been selected as follows: diameter $D=2 \mathrm{~nm}$, thickness $t=0.34 \mathrm{~nm}$, length $L=100 \mathrm{~nm}$, nominal Young's modulus $E_{0}=1 \mathrm{TPa}$ and mass density $\rho=2300 \mathrm{~kg} \mathrm{~cm}^{-3}$. An exponential form has been assumed for the distance-decaying function governing the long-range forces in Eq. (1), i.e.:

$g(x, \xi)=\exp \left(-\frac{|x-\xi|}{l_{0}}\right)$

where $l_{0}$ denotes the internal length material scale defining the size of the so-called influence distance, namely the maximum distance beyond which $g(x, \xi)$ and therefore the long-range interactions become negligible. The material constant $c_{q}$ in Eq. (1) has been set equal to [32]: chosen coincident with the autocorrelation function $R_{\tilde{B} \tilde{B}}(x, \xi) \equiv R_{\tilde{B} \tilde{B}}(|x-\xi|)$ characterizing the homogeneous zero-mean Gaussian random field $\tilde{B}(x)$ (see Eq. (41)) in the context of the

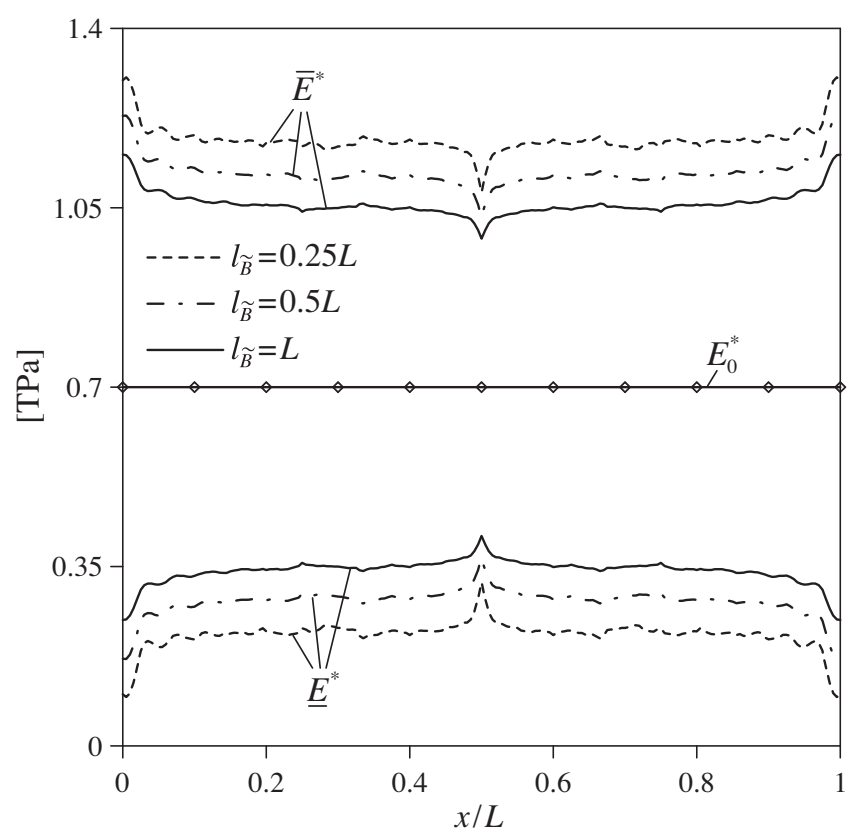

Fig. 2. Region of the interval Young's modulus $E^{* I}(x / L)$ along the $1 \mathrm{D}$ solid for different values of the parameter $l_{\tilde{B}}$ governing the spatial dependency of the interval field (see Eq. (62)). 
stochastic modelling of the uncertain material property. In particular, the following exponential function has been assumed:

$\Gamma_{B}(x, \xi) \equiv R_{\tilde{B} \tilde{B}}(|x-\xi|)=\sigma_{\tilde{B}}^{2} \exp \left(-\frac{|x-\xi|}{l_{\tilde{B}}}\right)$

where $\sigma_{\tilde{B}}^{2}=0.05$ and $l_{\tilde{B}}$ denotes the correlation length herein taken variable to investigate the effects of spatial correlation on the response. The function $\Gamma_{B}(x, \xi) \equiv R_{\tilde{B} \tilde{B}}(|x-\xi|)$ has been decomposed by applying Eq. (12) (or equivalently Eq. (43)) retaining $N=12$ $(N=M)$ terms. By comparing Eqs. (19) and (62), it is found that $\sigma_{\tilde{B}}^{2} \equiv C_{B}^{2}$ and $l_{\tilde{B}} \equiv l_{B}$.

Both the interval and stochastic integro-differential equilibrium equations in Eqs. (24) and (47) have been discretized by the finite difference method using a uniform grid with 200 subdivisions.
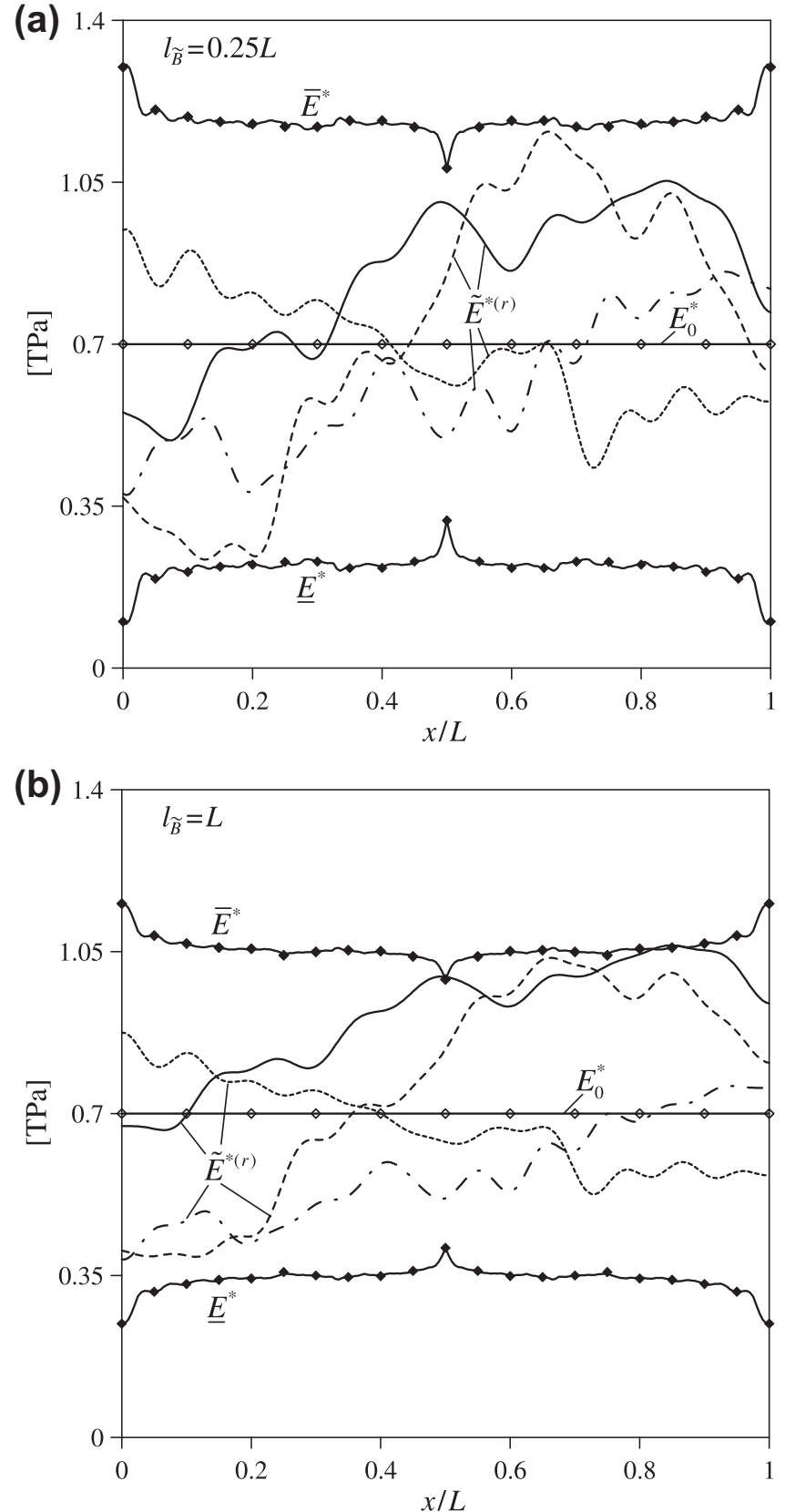

Fig. 3. Lower bound and upper bound of the interval field $E^{* I}(x / L)$ along the 1D solid contrasted to samples $\tilde{E}^{*(r)}(x / L),(r=1,2,3,4)$, of the homogeneous Gaussian random field $\tilde{E}^{*(r)}(x / L)$ : (a) $l_{\tilde{B}}=0.25 L$; (b) $l_{\tilde{B}}=L$.
As a first step, the consistency of the proposed definition of the interval field has been scrutinized. Fig. 2 displays the upper bound (UB) and lower bound (LB) of the Young's modulus interval field $E^{* I}(x)$ (see Eqs. $\left.(17 \mathrm{a}, \mathrm{b})\right)$ for three different values of the correlation length $l_{\tilde{B}}$ as well as the midpoint value $E_{0}^{*}=\beta_{1} E_{0}$ taken constant along the bar. As expected, the radius of the interval field is affected by the parameter $l_{\tilde{B}}$ governing the spatial dependency through the deterministic function $\Gamma_{B}(x, \xi)$ defined in Eq. (62). Specifically, the smaller the value of $l_{\tilde{B}}$ the larger the amplitude of the Young's modulus region. Conversely, as larger values of the parameter $l_{\tilde{B}}$ are considered, the deviation amplitude of the interval field $E^{* I}(x)$ decreases. As outlined in Section 3 , the limit case $l_{\tilde{B}} \rightarrow \infty$ corresponds to the total dependency condition in which the uncertain property is described by a single interval variable constant over the whole CNT domain $[0, L]$. It is argued, therefore, that the assumption of total dependency may lead to a serious underestimation of the bounds of the interval Young's modulus.
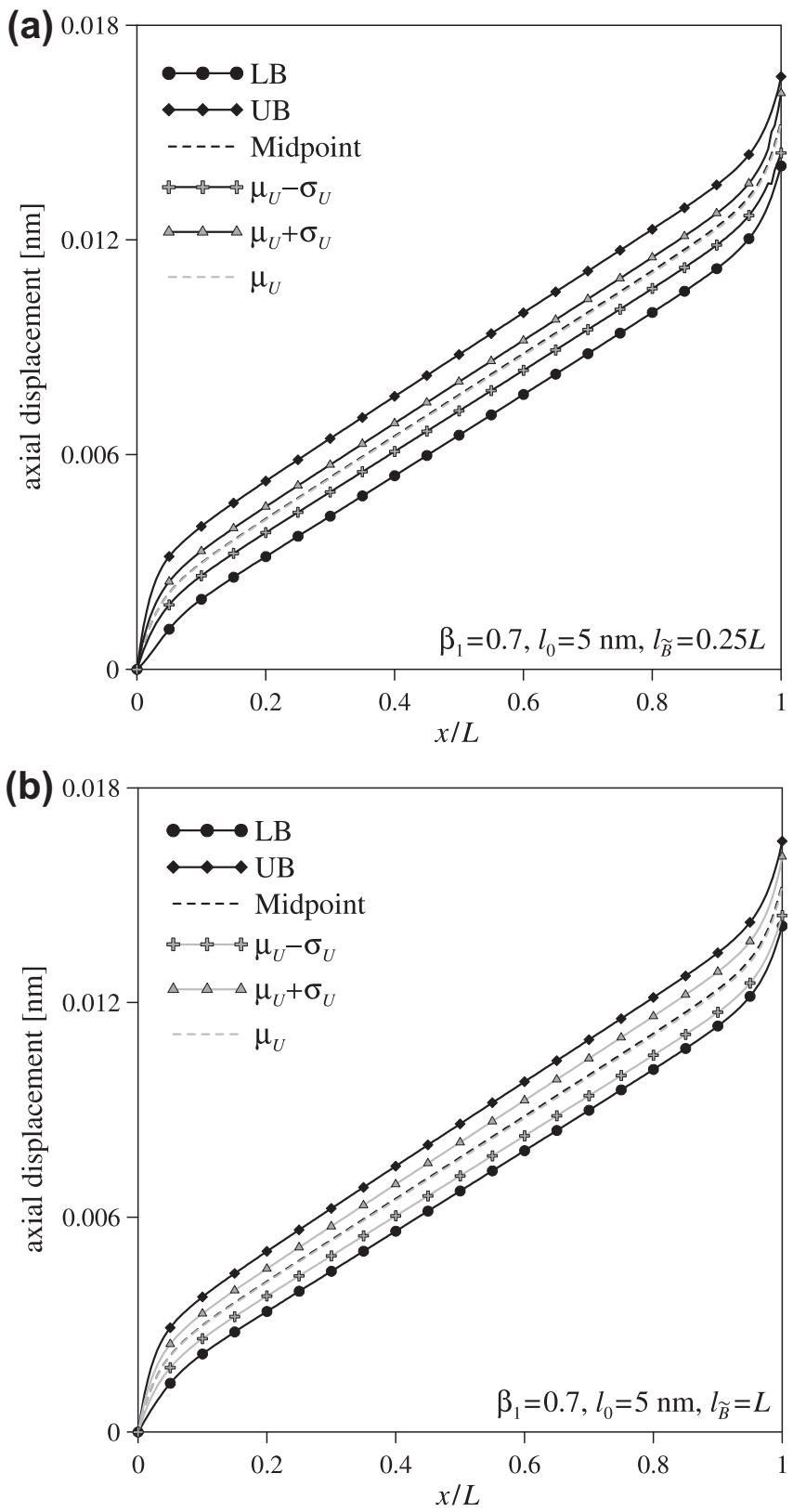

Fig. 4. Comparison between the interval and stochastic $\left(\mu_{U} \pm \sigma_{U}\right)$ regions of the axial displacement field along the non-local elastic bar: (a) $l_{\tilde{B}}=0.25 L$; (b) $l_{\tilde{B}}=L$. 
In Fig. 3, the bounds of the Young's modulus interval field, $E^{* I}(x)$, along with some samples $\tilde{E}^{*(r)}(x)$ of the corresponding homogeneous Gaussian random field, $\tilde{E}^{*}(x)$, for two different values of the correlation length $l_{\tilde{B}}$, are plotted. The region of the interval field almost encloses the samples of the random Young's modulus, consistently with the meaning of the interval model.

Within the context of the proposed definition of the Young's modulus interval field, the LB and UB of the interval displacement along the non-local bar under tension have been computed by applying the closed-form expressions derived in Section 4. Similarly, the mean-value, $\mu_{U}(x)$, and the standard deviation, $\sigma_{U}(x)$, of the stochastic displacement field in the case of randomly varying Young's modulus have been determined by using the explicit expressions presented in Section 5. The region of the non-local displacement field provided by the proposed improved interval analysis has been compared with the confidence interval of the stochastic response bounded by the values $\mu_{U}(x)-k \sigma_{U}(x)$ and $\mu_{U}(x)+k \sigma_{U}(x), k$ being a positive integer. In Fig. 4 , the interval region is compared with the stochastic one for $k=1$, considering different values of the correlation length $l_{\tilde{B}}$ in Eq. (62). As expected, both the interval and stochastic responses are affected by the correlation length $l_{\tilde{B}}$. It can be observed that the confidence interval involved in stochastic analysis turns out to be tighter than the region of the displacement field obtained via interval analysis for all values of the correlation length $l_{\tilde{B}}$ herein considered.

To gain a deeper insight into the effects of the interval and stochastic modeling of the uncertain Young's modulus on the non-local response of the CNT under tension, in Fig. 5 the radius of the interval displacement, $\Delta u(x)$, is compared with the standard deviation, $\sigma_{U}(x)$, of the random response for different values of the parameter $l_{\tilde{B}}$. It can be seen that the condition $\Delta u(x)>\sigma_{U}(x)$ holds. Furthermore, the correlation length $l_{\tilde{B}}$ has a different influence on the interval and stochastic responses. Indeed, as the parameter $l_{\tilde{B}}$ decreases, the radius of the interval displacement, $\Delta u(x)$, increases, while smaller values of the standard deviation of the random response, $\sigma_{U}(x)$, are obtained.

Similar considerations can be drawn by investigating the dispersion of the interval and random responses around the corre-

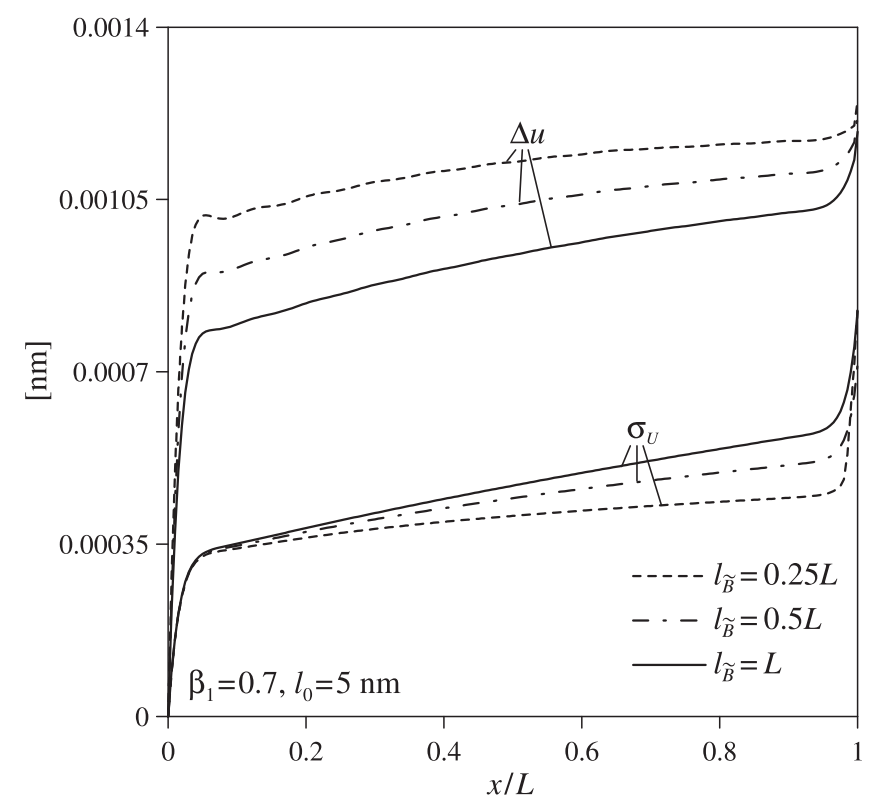

Fig. 5. Radius of the interval displacement field, $\Delta u(x / L)$, and standard deviation of the random displacement field, $\sigma_{U}(x / L)$, along the non-local bar under tension for different values of the parameter $l_{\tilde{B}}$ governing the spatial dependency of the interval and stochastic Young's modulus (see Eq. (62)).

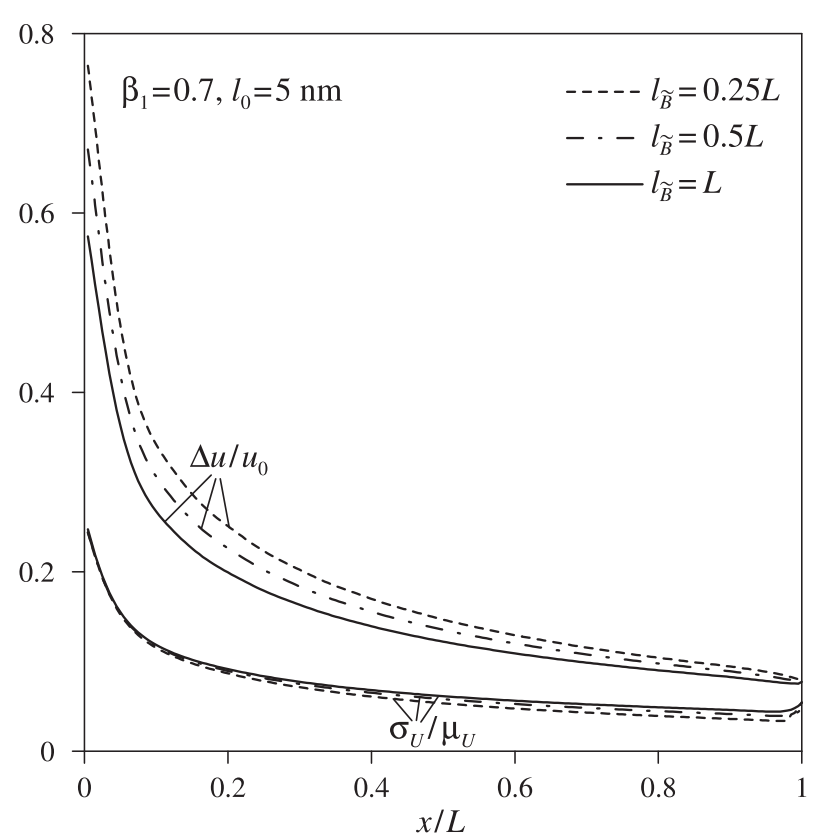

Fig. 6. Coefficient of interval uncertainty, $\Delta u / u_{0}$, of the interval displacement field and coefficient of variation, $\sigma_{U} / \mu_{U}$, of the random displacement field along the nonlocal bar under tension for different values of the parameter $l_{\tilde{B}}$ governing the spatial dependency of the interval and stochastic Young's modulus (see Eq. (62)).

sponding midpoint and mean-values, $u_{0}(x)$ and $\mu_{U}(x)$. Appropriate measures of such dispersion are the well-known coefficient of variation for the random displacement field, $\sigma_{U}(x) / \mu_{U}(x)$, and its non-probabilistic counterpart given by the so-called coefficient of interval uncertainty, $\Delta u(x) / u_{0}(x)$. Fig. 6 shows that the interval response exhibits a larger dispersion than the stochastic one for all values of the correlation length $l_{\tilde{B}}$, in agreement with the comparison between the regions of the interval and stochastic displacements plotted in Fig. 4. Furthermore, it is noted that the extreme assumption of total spatially dependent interval field corresponding to $l_{\tilde{B}} \rightarrow \infty$ would lead to an underestimation of the interval response deviation. Indeed, both Figs. 5 and 6 show that, as $l_{\tilde{B}}$ increases, the deviation of the interval displacement with respect to the midpoint value decreases.

\section{Conclusions}

The analysis of one-dimensional heterogeneous non-local elastic solids with fluctuating Young's modulus of the material has been addressed. According to a recently proposed mechanicallybased approach, non-local effects have been described as longrange interactions between non-adjacent volume elements. Longrange forces depend on the product of interacting masses, as well as on their relative displacements by means of a proper materialdependent distance-decaying function. Besides the traditional modeling of Young's modulus fluctuation as a homogeneous Gaussian random field, a non-probabilistic approach has been applied to describe the material property variability along the onedimensional solid. To this aim, a novel definition of the interval field concept has been presented which allows to account for the dependency between interval values at various locations by introducing a deterministic symmetric non-negative bounded function playing the same role of the autocorrelation function in random field theory. The main novelty of the proposed interval field model consists in its decomposition as superposition of interval functions through the use of a proper extension of the Karhunen-Loève expansion in conjunction with the improved interval analysis, re- 
cently proposed by the first two authors to limit the overestimation due to the dependency phenomenon. Such decomposition allows to build formally analogous integro-differential equilibrium equations within the interval and stochastic settings. Based on this analogy, after performing a finite difference discretization, an efficient procedure is proposed to derive approximate explicit expressions of the bounds of the interval displacement field and of the mean-value and variance of the random response. Furthermore, a meaningful mechanical interpretation of the 1D heterogeneous solid with long-range interactions in presence of interval or random Young's modulus fluctuations has been provided.

Numerical results have demonstrated the consistency of the novel definition of the interval field as well as the accuracy of the proposed approximate closed-form solutions.

\section{Appendix A. Basic elements of interval algebra}

Interval algebra is an elegant tool to solve practical problems with inequalities like, for instance, those occurring in presence of approximate numbers, error bounds, uncertain experimental data and so on. Then, the purpose of interval analysis is to provide upper and lower bounds on the effects all such errors and uncertainties have on a computed quantity. Furthermore, the major focus of interval analysis is to develop practical interval algorithms that produce sharp (as narrow as possible) or nearly sharp bounds on the solution of numerical computing problems $[6-8,41]$. The history of interval analysis could go all the way back to Archimedes who defined the transcendental number $\pi$ by an interval: 30/ $71<\pi<3 / 7[4,5,8]$. He derived such bounds showing that the number $\pi$ belongs to the interval obtained by approximating the circle with the inscribed and circumscribed 96-side regular polygons.

Recent developments in interval arithmetic are mainly based on the book of Moore [6], who introduced the so-called "ordinary" interval algebra as well as the interval vectors and matrices with their first non-trivial applications.

In this Appendix, the fundamentals of interval algebra are briefly summarized and some basic notations are introduced.

While the field of real numbers is denoted by $\mathbb{R}$, the field of all closed real interval numbers is denoted by $\mathbb{Q R}$. In particular, a subset of $\mathbb{R}$ of the form:

$x^{I} \equiv[x] \triangleq[\underline{x}, \bar{x}]=\{x \mid \underline{x} \leqslant x \leqslant \bar{x}, \quad x \in \mathbb{R}\}$

is called a closed real interval or an interval if no confusion arises. In writing Eq. (A.1.1), the set-builder notation $\{x \mid P(x)\}$ is adopted which defines $x^{I}$ as "the set of all elements $x$ such that the proposition $P(x)$ holds". The apex $I$ denotes an interval variable $\in \mathbb{R} \mathbb{R}$ and $\underline{x}$ and $\bar{x}$ define the lower and upper bounds of the interval, respectively. Alternatively, an interval $x^{I}$ could be represented by its midpoint (or mean), $x_{0}$, and by the deviation (or half-width also simply termed width), $\Delta x$, i.e.

$x_{0}=\frac{\bar{x}+\underline{x}}{2} ; \quad \Delta x=\frac{\bar{x}-\underline{x}}{2}$.

Denoting by $x^{I}, y^{I}, z^{I} \in \mathbb{R} \mathbb{R}$ three closed bounded intervals, the basic interval operations are listed below:

$x^{I}+y^{I}=[\underline{x}+y, \bar{x}+\bar{y}] ;$

$x^{I}-y^{I}=[\underline{x}-\bar{y}, \bar{x}-\underline{y}] ;$

$x^{I} \times y^{I}=[\min (\underline{x y}, \underline{x} \bar{y}, \bar{x} y, \bar{x} \bar{y}), \max (\underline{x y}, \underline{x y}, \bar{x} y, \overline{x y})] ;$

(A.1.3a-d)

$x^{I} / y^{I}=[\underline{x}, \bar{x}] \times[1 / \bar{y}, 1 / y]$ if $0 \notin y^{I}$.

Furthermore, two arithmetic expressions which are equivalent in real arithmetic are also equivalent in interval arithmetic when every variable occurs only once on each side. Therefore, the following properties are in agreement with real arithmetic:

$$
\left.\begin{array}{cc}
x^{I}+y^{I}=y^{I}+x^{I} ; x^{I} \times y^{I}=y^{I} \times x^{I} & \text { (commutativity) } \\
\left(x^{I}+y^{I}\right) \pm z^{I}=x^{I}+\left(y^{I} \pm z^{I}\right) \\
\left(x^{I} \times y^{I}\right) \times z^{I}=x^{I} \times\left(y^{I} \times z^{I}\right)
\end{array}\right\} \quad \text { (associativity) }
$$

while the following ones are in disagreement:

$$
\left.\begin{array}{ll}
x^{I} \times\left(y^{I}+z^{I}\right) \subseteq x^{I} y^{I}+x^{I} z^{I} & \text { (subdistributivity) } \\
x^{I}-y^{I} \subseteq\left(x^{I}+z^{I}\right)-\left(y^{I}+z^{I}\right), \quad 0 \in x^{I}-x^{I} \\
x^{I} / y^{I} \subseteq\left(x^{I} z^{I}\right) /\left(y^{I} z^{I}\right), \quad 1 \in x^{I} / x^{I}
\end{array}\right\} \quad \text { (subcancellation) }
$$

Notice that the relaxation of distributivity and cancellation properties of the traditional arithmetic to the subdistributivity and subcancellation properties of the interval arithmetic is due to the socalled dependency phenomenon. This phenomenon arises because different occurrences of a single interval variable in an expression are treated as independent variables. For example, if the interval $x^{I}=[0,1]$ is subtracted from itself, the interval $[-1,1]$ is obtained as result and not $[0,0]$, as in the traditional algebra. Another typical example of the dependence phenomenon is the evaluation of the bounds of the function $f\left(x^{I}\right)=x^{I} /\left(1+x^{I}\right)$ in which the interval variable $x^{I}$ appears twice $[5,7,8,41]$. The dependency problem can be eliminated if the previous function is rewritten in the following equivalent form $\hat{f}\left(x^{I}\right)=1 /\left[1+\left(1 / x^{I}\right)\right]$ where the interval variable appears only once. Indeed, the two functions $f(x)=x /(1+x)$ and $\hat{f}(x)=1 /[1+(1 / x)]$, which are equivalent in the classical real arithmetic, $x \in \mathbb{R}$, turn out to be different in the framework of interval analysis, $x \in \mathbb{R}$. As an example, if we assume $x \in[0,1]$, the first interval function $f\left(x^{I}\right)$ furnishes the interval $[0,1]$, which overestimates the actual interval $[0,1 / 2]$ obtained by means of the second expression of the interval function $\hat{f}\left(x^{I}\right)$. The two simple examples described above show that the subcancellation and subdistributivity properties give an overestimation of the interval width when the same variable $x^{I}$ appears twice in the same expression.

An $n$-dimensional interval vector, $\mathbf{x}^{I} \in \mathbb{\mathbb { R } ^ { n }}$ is an ordered $n$-tuple of intervals $\left(x_{1}^{I}, x_{2}^{I}, \ldots, x_{n}^{I}\right)$. If two vectors $\underline{\mathbf{x}}, \overline{\mathbf{x}} \in \mathbb{R}^{n}$ satisfy the condition $\underline{\mathbf{x}} \leqslant \overline{\mathbf{x}}$ (or equivalently $\underline{x}_{j} \leqslant \bar{x}_{j}$ ), an interval vector, $\mathbf{x}^{I} \in \mathbb{R} \mathbb{R}^{n}$, is generally identified with the (nonempty) set of vectors of the form:

$\mathbf{x}^{I} \triangleq[\underline{\mathbf{x}}, \overline{\mathbf{x}}]=\left\{\mathbf{x} \mid \underline{\mathbf{x}} \leqslant \mathbf{x} \leqslant \overline{\mathbf{x}}, \quad x_{j} \in \mathbb{R}\right\}$.

With suitable modifications, many of the notions for ordinary intervals can be extended to interval vectors. An interval vector $\mathbf{x}^{I}$ could be also represented by its midpoint (or mean) vector, $\mathbf{x}_{0}$, and by the deviation (radius or half-width also simply termed width) vector, $\Delta \mathbf{x}$, i.e.

$\mathbf{x}_{0}=\frac{1}{2}(\overline{\mathbf{x}}+\underline{\mathbf{x}}) ; \quad \Delta \mathbf{x}=\frac{1}{2}(\overline{\mathbf{x}}-\underline{\mathbf{x}}) \Longleftrightarrow \underline{\mathbf{x}}=\mathbf{x}_{0}-\Delta \mathbf{x} ; \quad \overline{\mathbf{x}}=\mathbf{x}_{0}+\Delta \mathbf{x}$.

(A.1.7.a-d)

Obviously, $\Delta \mathbf{x}$ is a nonnegative vector, that is all its elements are positive numbers. An $m \times n$ matrix is an $m \times n$ interval matrix, $\mathbf{A}^{I} \in \mathbb{\mathbb { R } ^ { m \times n }}$, if its entries $a_{j k}^{I}=\left[\underline{a}_{j k}, \bar{a}_{j k}\right](j=1, \ldots, m ; k=1, \ldots, n)$ are intervals. Alternatively, the interval matrix $\mathbf{A}^{I}$ can be defined as:

$\mathbf{A}^{I} \triangleq[\underline{\mathbf{A}}, \overline{\mathbf{A}}]=\left\{\mathbf{A} \mid \underline{\mathbf{A}} \leqslant \mathbf{A} \leqslant \overline{\mathbf{A}}, \quad a_{j k} \in \mathbb{R}\right\}$

where $\underline{\mathbf{A}}$ and $\overline{\mathbf{A}}$ are the lower and upper bounds matrices. The following properties hold for matrix's addition and subtraction:

$\mathbf{A}+\mathbf{B}=[\underline{\mathbf{A}}+\underline{\mathbf{B}}, \overline{\mathbf{A}}+\overline{\mathbf{B}}]$

$\mathbf{A}-\mathbf{B}=[\underline{\mathbf{A}}-\overline{\mathbf{B}}, \overline{\mathbf{A}}-\underline{\mathbf{B}}]$

$\mathbf{A}^{I}+\mathbf{B}^{I}=\mathbf{B}^{I}+\mathbf{A}^{I}$

(commutativity)

(A.1.9a-d)

$\mathbf{A}^{I}+\left(\mathbf{B}^{I}+\mathbf{C}^{I}\right)=\left(\mathbf{A}^{I}+\mathbf{B}^{I}\right)+\mathbf{C}^{I} ; \quad$ (associativity) 
Notice that the product of two or more interval matrices may be subjected to interval dependency $[7,8,41,42]$. It follows that the associative and distributive properties may not hold for the interval matricial product $\mathbf{C}^{I}=\mathbf{A}^{I} \times \mathbf{B}^{I}$. The dependency phenomenon occurs because the interval arithmetic does not assume that the same point elements are chosen from the interval elements of the left matrix $\mathbf{A}^{I}$ in forming the sets comprising the different columns of the product interval matrix $\mathbf{C}^{I}$. This is similar to interval dependency in scalar expressions. Indeed, each interval element of the left matrix $\mathbf{A}^{I}$ is an interval variable which occurs multiple times, once for each column, in forming the columns of the interval product matrix $\mathbf{C}^{I}$.

\section{Appendix B. Solutions of a set of linear interval equations}

The Finite Element Method (FEM) is one of the most celebrated numerical methods for solving differential equations with enormous applications in different fields of sciences and engineering. In the static analysis of structures, the FEM performs the solution of a differential problem through the solution of a set of algebraic equations. In several structural problems, different sources of uncertainties may affect the mathematical model. It follows that the associated set of governing equations is also affected by uncertainties. If the uncertainties are modelled by interval variables, the structural response is obtained as solution of a set of linear interval algebraic equations. Since the main drawback of the interval analysis is the dependency phenomenon, researches in the framework of structural analysis have focused on two major problems: the first one is how to obtain solutions for the resulting set of linear interval equations with reasonable bounds on the system response that make sense from a practical point of view, or in other words, with the least possible overestimation of their bounding intervals; the second problem is how to obtain reasonable bounds on the derived quantities that are functions of the system response [43].

To gain further insight into these problems, we recall that in the framework of interval analysis an interval matrix $\mathbf{B}^{I}=[\underline{\mathbf{B}}, \overline{\mathbf{B}}]$, satisfying $\left(\mathbf{A}^{I}\right)^{-1} \subseteq[\underline{\mathbf{B}}, \overline{\mathbf{B}}]$, is called an enclosure of the inverse interval matrix [44]. This matrix can be evaluated if and only if the square matrix $\mathbf{A}$ is regular, that is a matrix for which each $\mathbf{A} \in \mathbf{A}^{I}$ is nonsingular. A set of linear interval equations with coefficient matrix $\mathbf{A}^{I} \in \mathbb{Q} \mathbb{R}^{n \times n}$ and right-hand side $\mathbf{b}^{I} \in \mathbb{\mathbb { R } ^ { n }}$ is defined as the family of linear equations

$\mathbf{A}^{I} \mathbf{x}^{I}=\mathbf{b}^{I}$.

The narrowest interval vector containing the solution set of Eq. (A.2.1) is called the interval hull of the solution. Then, the interval hull of the solution of linear interval equations is an interval vector, $\mathbf{x}^{I(H)} \in \mathbf{X}^{I}$, that contains the solution set and has the narrowest possible interval components. In the framework of the "ordinary" interval analysis, there are two types of methods for the numerical solution of such problems [8,42]: direct and iterative methods. Direct methods, such as Gaussian elimination (with or without various "pivoting" schemes), can produce exact results in a finite number of arithmetic operations if the matrix is regular and if infinite precision arithmetic is used. Iterative methods produce a sequence of approximate solutions which converge to the unique solution. One of the best known methods for obtaining very sharp enclosures of interval linear set of equations is the iterative method developed in the work [45]. However, the main drawbacks of these methods are their complexity, which often produces an overestimation of the interval vector solution, and their poor flexibility for specific structural problems in which the interval matrix of coefficients takes a very particular expression (see e.g. Eq. (27)). To overcome these limitations, two new approaches have been recently proposed: the improved interval analysis [15] and the parameterized interval analysis $[47,48]$. In the present paper, the first approach is adopted which requires the following main steps $[15,39,46]$ : (i) the introduction of the so-called extra unitary interval (EUI) variable; (ii) the expansion of the interval coefficient matrix in a series of rank-one interval matrices. The use of the EUI variable, for monotonic interval functions, drastically reduces the effects of the dependency phenomenon. Indeed, the EUI variable, associated to an interval parameter, follows the properties (4). Such properties are different from the ones obtained for the "ordinary" unitary symmetric interval $e^{I} \triangleq[-1,+1]$ by applying the rules (A.1.3) of the "ordinary" interval algebra, which read:

$$
\begin{aligned}
& e^{I}-e^{I}=[-2,+2] ; e^{I} \times e^{I}=[-1,+1] \\
& e^{I} / e^{I} \quad \text { does not exist because } 0 \in[-1,+1] ; \\
& x_{i} e^{I} \pm y_{i} e^{I}=\left[-x_{i}-y_{i}, x_{i}+y_{i}\right] \\
& x_{i} e^{I} \times y_{i} e^{I}=\left[-x_{i} y_{i}, x_{i} y_{i}\right]
\end{aligned}
$$

As an example, by adopting the EUI variable the two functions $f\left(x^{I}\right)=x^{I} /\left(1+x^{I}\right)$ and $\hat{f}\left(x^{I}\right)=1 /\left[1+\left(1 / x^{I}\right)\right]$, unlike "ordinary" interval algebra (see Appendix $A$ ), give the same result in the interval $x \in[0,1]$, as it should be.

The second step involved in the improved interval analysis, say the expansion of the interval coefficient matrix, produces an approximate, but very accurate, explicit expression of the inverse of the interval matrix (see Section 4). So operating, the overestimation due to the dependency phenomenon is drastically reduced.

Finally, let us observe that the definition (11) of the symmetric non-negative function, $\Gamma_{B}(x, \xi)$ is strictly connected to the use of the improved interval analysis. Indeed, by applying the "ordinary" interval algebra, the product in Eq. (11) gives a different result:

$$
\begin{aligned}
& B^{I}(x) B^{I}(\xi)=[\min (\underline{B}(x) \underline{B}(\xi), \underline{B}(x) \bar{B}(\xi), \bar{B}(x) \underline{B}(\xi), \bar{B}(x) \bar{B}(\xi)), \\
& , \max (\underline{B}(x) \underline{B}(\xi), \underline{B}(x) \bar{B}(\xi), \bar{B}(x) \underline{B}(\xi), \bar{B}(x) \bar{B}(\xi))]
\end{aligned}
$$

\section{References}

[1] Kleiber M, Hien HD. The stochastic finite element method: basic perturbation technique and computer implementation. Chichester: John-Wiley \& Sons: 1992.

[2] Ghanem RG, Spanos PD. Stochastic finite elements: a spectral approach. New York, USA: Springer-Verlag; 1991.

[3] Moens D, Vandepitte D. A survey of non-probabilistic uncertainty treatment in finite element analysis. Comput Methods Appl Mech Eng 2005;194:1527-55.

[4] Ben-Haim Y, Elishakoff I. Convex models of uncertainty in applied mechanics. Amsterdam: Elsevier; 1990.

[5] Elishakoff I, Ohsaki M. Optimization and anti-optimization of structures under uncertainty. London: Imperial College Press; 2010.

[6] Moore RE. Interval analysis. Englewood Cliffs: Prentice-Hall; 1966.

[7] Alefeld G, Herzberger J. Introduction to interval computations. New York: Academic Press; 1983.

[8] Moore RE, Kearfott RB, Cloud MJ. Introduction to interval analysis. Philadelphia: SIAM; 2009.

[9] Chen SH, Lian HD, Yang XW. Interval static displacement analysis for structures with interval parameters. Int J Numer Methods Eng 2002:53:393-407.

[10] Qiu ZP, Wang XJ. Parameter perturbation method for dynamic responses of structures with uncertain-but-bounded parameters based on interval analysis. Int J Solids Struct 2005;4:4958-70.

[11] Muscolino G, Sofi A. Stochastic response of structures with uncertain-butbounded parameters. In: Proceedings of IMECE2009, November 13-19, Lake Buena Vista, Florida, USA, 2009.

[12] Muscolino G, Sofi A. Response statistics of linear structures with uncertainbut-bounded parameters under gaussian stochastic input. Int J Struct Stab Dyn 2011;11:1-30

[13] Muscolino G, Sofi A. Response of structural systems with uncertain-butbounded parameters under stationary stochastic input via interval analysis. In: EURODYN 2011. De Roeck G, Degrande G, Lombaert G, Muller G, editors. Proceedings of the 8th International Conference on Structural Dynamics, Leuven, Belgium; 4-6 July 2011. p. 3016-3023.

[14] Muscolino G, Sofi A. Stochastic analysis of structures with uncertain-butbounded parameters. In: Deodatis G, Spanos PD, editors. Computational stochastic mechanics. Singapore: Research Publishing; 2011. p. 415-27. 
[15] Muscolino G, Sofi A. Stochastic analysis of structures with uncertain-butbounded parameters via improved interval analysis. Probab Eng Mech 2012;28:152-63.

[16] Moens D, De Munck M, Desmet W, Vandepitte D. Numerical dynamic analysis of uncertain mechanical structures based on interval fields. In: Belyaev AK Langley RS. editors. IUTAM Symposium on the Vibration Analysis of Structures with Uncertainties, Springer, Dordrecht; 2011, p. 71-83.

[17] Verhaeghe W, Desmet W, Vandepitte D, Joris I, Seuntjens P, Moens D. Application of interval fields for uncertainty modelling in a geohydrological case. In: Papadrakakis M, Fragiadakis M, Plevris V, editors. Compdyn 2011-3 ECCOMAS Thematic Conference, Corfu, Greece; 25-28 May 2011.

[18] Muhanna RL, Mullen RL. Uncertainty in mechanics problems-interval-based approach. J Eng Mech-ASCE 2001;127:557-66.

[19] Kroner E. Elasticity theory of materials with long-range cohesive forces. Int J Solids Struct 1967;3:731-42.

[20] Eringen AC. Theory of micropolar continua. In: Huang TC, Johnson MW, editors. Developments in mechanics. New York: John Wiley; 1967.

[21] Kunin IA. The theory of elastic media with microstructure and the theory of dislocations. In: Kröner E, editor. Mechanics of generalized continua. New York: Springer-Verlag; 1968.

[22] Eringen AC. Linear theory of nonlocal elasticity and dispersion of plane waves. Int J Eng Sci 1972;10:425-35.

[23] Eringen AC. Screw dislocation in non-local elasticity. J Phys D 1977;10:671-8.

[24] Aifantis EC. Gradient effects at macro micro and nano scales. J Mech Behav Mater 1994;5:355-75.

[25] Aifantis EC. Update on a class of gradient theories. Mech Mater 2003;35:259-80.

[26] Di Paola M, Zingales M. Long-range cohesive interactions of non-local continuum faced by fractional calculus. Int J Solids Struct 2008;45(21): 5642-59.

[27] Di Paola M, Failla G, Zingales M. Physically-based approach to the mechanics of strong non-local linear elasticity theory. J Elast 2009;97:103-30.

[28] Di Paola M, Pirrotta A, Zingales M. Mechanically-based approach to non-local elasticity: variational Principles. Int J Solids Struct 2010;47:539-48.

[29] Zingales M. Wave Propagation in 1D elastic solids in presence of long-range central interactions. J Sound Vib 2011;330:3973-89.

[30] Sobczyk K, Kirkner DJ. Stochastic modeling of microstructures. Boston: Birkäuser; 2001.

[31] Aifantis EC, Frantziskonis G. On the stochastic interpretation of gradientdependent constitutive equations. Eur J Mech A/Solids 2002;21:589-96.
[32] Di Paola M, Sofi A, Zingales M. Stochastic analysis of 1D heterogeneous solids with long-range interactions. J Multiscale Comput Eng 2011;9(4):379-94.

[33] Hansen ERA. Generalized interval arithmetic. In: Nicket K, editor. Interval Mathematics, Lect Notes Computer Science. vol. 29; 1975. p. 7-18.

[34] Comba JLD, Stolfi J. Affine arithmetic and its applications to computer graphics. Anais do VI Simposio Brasileiro de Computaao Grafica e Processamento de Imagens (SIBGRAPI'93"), Recife (Brazil), October 9-18, 1993.

[35] Stolfi J, de Figueiredo LH. An introduction to affine arithmetic. TEMA Tend Mat Apl Comput 2003:4:297-312.

[36] Nedialkov NS, Kreinovich V, Starks SA. Interval arithmetic, affine arithmetic, Taylor series methods: why, what next? Numer Algorithms 2004;37:325-36.

[37] Rohn J. Interval solution of linear interval equations. Appl Math 1990;35:220-4.

[38] Muscolino G, Sofi A. Bounds for the stationary stochastic response of truss structures with uncertain-but-bounded parameters. Mech Syst Signal Process 2012. http://dx.doi.org/10.1016/j.ymssp.2012.06.016.

[39] Muscolino G, Sofi A. Explicit solutions for the static and dynamic analysis of discretized structures with uncertain parameters. In: Computational Methods for Engineering Science. Topping BHV, editor. Saxe-Coburg Publications, Stirlingshire, Scotland, vol. 30, Chapter 3: 47-73; 2012.

[40] Kamiński M. A generalized version of the perturbation-based stochastic finite difference method for elastic beams. J Theor Appl Mech 2009;47(4):957-75.

[41] Hansen E, Walster GW. Global optimization using interval analysis. New York: Marcel Dekker, Inc. and Sun Microsystems, Inc.; 2004. 2nd ed., Revised and Expanded.

[42] Neumaier A. Interval methods for systems of equations. Cambridge, UK: Cambridge University Press; 1990.

[43] Rama Rao MV, Mullen RL, Muhanna RL. A new interval finite element formulation with the same accuracy in primary and derived variables. Int J Reliab Saf 2011;5(3/4):336-57.

[44] Rohn J. Inverse interval matrix. SIAM J Numer Anal 1993;30:864-70.

[45] Neumaier A, Pownuk A. Linear systems with large uncertainties, with applications to truss structures. Reliable Comput 2007;13:149-72.

[46] Impollonia N, Muscolino G. Interval analysis of structures with uncertain-butbounded axial stiffness. Comput Methods Appl Mech Eng 2011;220:1945-62.

[47] Elishakoff I, Miglis Y. Novel parameterized intervals may lead to sharp bounds. Mech Res Commun 2012;44:1-8.

[48] Elishakoff I, Miglis Y. Overestimation-free computational version of interval analysis. Int J Comput Methods Eng Sci Mech 2012;13:319-28. 